\title{
Unimodular Hyperbolic Triangulations: Circle Packing and Random Walk
}

\section{Omer Angel Tom Hutchcroft Asaf Nachmias Gourab Ray}

\begin{abstract}
We show that the circle packing type of a unimodular random plane triangulation is parabolic if and only if the expected degree of the root is six, if and only if the triangulation is amenable in the sense of Aldous and Lyons [1]. As a part of this, we obtain an alternative proof of the Benjamini-Schramm Recurrence Theorem [19].

Secondly, in the hyperbolic case, we prove that the random walk almost surely converges to a point in the unit circle, that the law of this limiting point has full support and no atoms, and that the unit circle is a realisation of the Poisson boundary. Finally, we show that the simple random walk has positive speed in the hyperbolic metric.
\end{abstract}

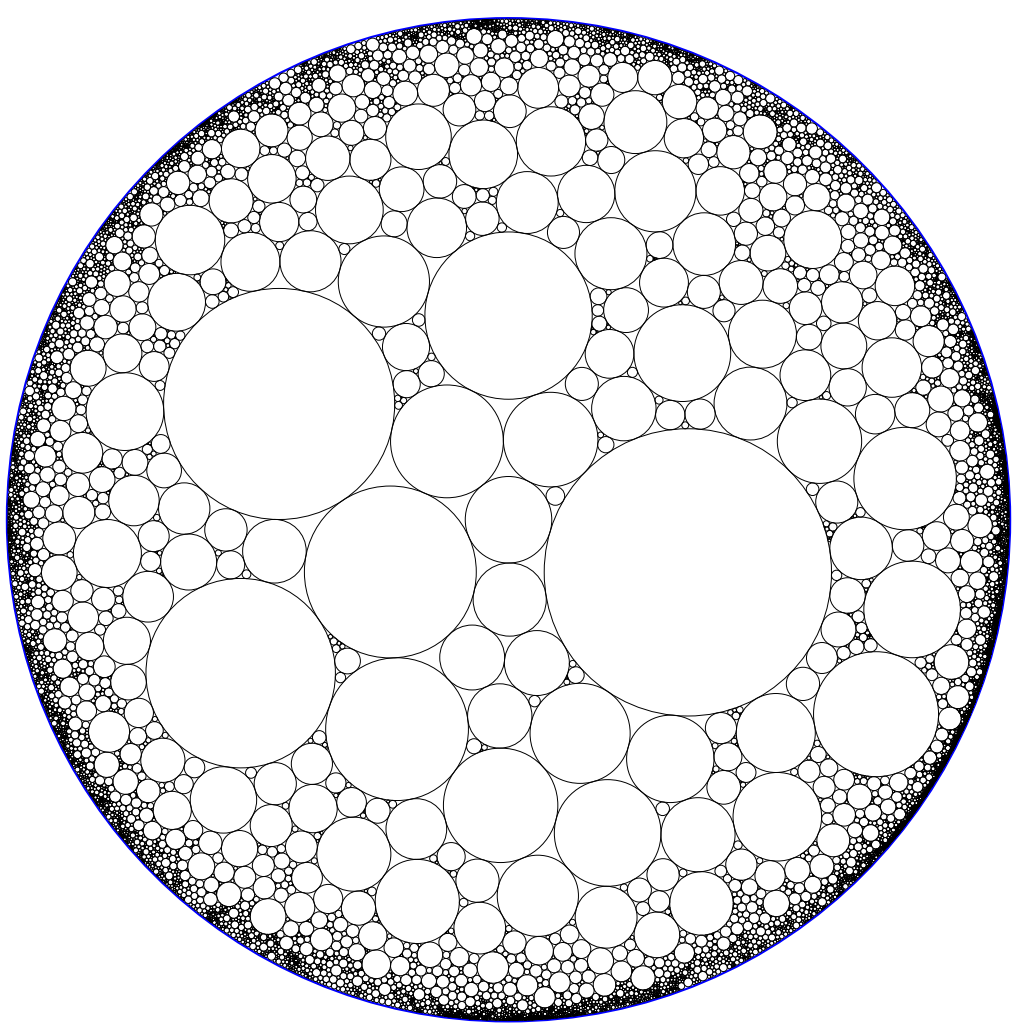

Figure 1: A circle packing of a random hyperbolic triangulation. 


\section{Introduction}

A circle packing of a planar graph $G$ is a set of circles with disjoint interiors in the plane, one for each vertex of $G$, such that two circles are tangent if and only if their corresponding vertices are adjacent in $G$. The Koebe-Andreev-Thurston Circle Packing Theorem [31, 42] states that every finite simple planar graph has a circle packing; if the graph is a triangulation (i.e. every face has three sides), the packing is unique up to Möbius transformations and reflections. He and Schramm $[27,28]$ extended this theorem to infinite, one-ended, simple triangulations, showing that each such triangulation admits a locally finite circle packing either in the Euclidean plane or in the hyperbolic plane (identified with the interior of the unit disc), but not both. See Section 3.4 for precise details. This result is a discrete analogue of the Uniformization Theorem, which states that every simply connected, non-compact Riemann surface is conformally equivalent to either the plane or the disc (indeed, there are deep connections between circle packing and conformal maps, see $[38,41]$ and references therein). Accordingly, a triangulation is called CP parabolic if it can be circle packed in the plane and CP hyperbolic otherwise.

Circle packing has proven instrumental in the study of random walks on planar graphs $[16,19,28,24]$. For graphs with bounded degrees, a rich theory has been established connecting the geometry of the circle packing and the behaviour of the random walk. Most notably, a one-ended, bounded degree triangulation is CP hyperbolic if and only if random walk on it is transient [28] and in this case it is also non-Liouville, i.e. admits non-constant bounded harmonic functions [16].

The goal of this work is to develop a similar, parallel theory for random triangulations. Particular motivations come from the Markovian hyperbolic triangulations constructed recently in [9] and [20]. These are hyperbolic variants of the UIPT [10] and are conjectured to be the local limits of uniform triangulations in high genus. Another example is the Poisson-Delaunay triangulation in the hyperbolic plane, studied in [18] and [15]. All these triangulations have unbounded degrees, rendering existing methods ineffective (for example methods used in $[4,16,28]$ ).

Indeed, in the absence of bounded degree the existing theory fails in many ways. For example, in a circle packing of a triangulation with bounded degrees, radii of adjacent circles have uniformly bounded ratios (a fact known as the Ring Lemma [37]). The absence of such a uniform bound invalidates important resistance estimates. This is not a mere technicality: one can add extra circles in the interstices of the circle packing of the triangular lattice to give the random walk drift in arbitrary directions. This does not change the circle packing type, but allows construction of a graph that is $\mathrm{CP}$ parabolic but transient or even non-Liouville. Indeed, the main effort in [24] was to overcome this sole obstacle in order to prove that the UIPT is recurrent. 
The hyperbolic random triangulations of [20] and [15] make up for having unbounded degrees by a different useful property: unimodularity (essentially equivalent to reversibility, see Sections 3.1 and 3.2). This allows us to apply probabilistic and ergodic arguments in place of the analytic arguments appropriate to the bounded degree case. Our first main theorem establishes a probabilistic characterisation of the $\mathrm{CP}$ type for unimodular random rooted triangulations, and connects it to the geometric property of invariant (non-)amenability, which we define in Section 3.3.

Theorem 1.1. Let $(G, \rho)$ be an infinite, simple, one-ended, ergodic unimodular random rooted planar triangulation. Then either

$\mathbb{E}[\operatorname{deg}(\rho)]=6$, in which case $(G, \rho)$ is invariantly amenable and almost surely CP parabolic,

or else

$\mathbb{E}[\operatorname{deg}(\rho)]>6$, in which case $(G, \rho)$ is invariantly non-amenable and almost surely CP hyperbolic.

This theorem can be viewed as a local-to-global principle for unimodular triangulations. That is, it allows us to identify the circle packing type and invariant amenability, both global properties, by calculating the expected degree, a very local quantity. For example, if $(G, \rho)$ is a simple, one-ended triangulation that is obtained as a local limit of planar graphs, then by Euler's formula and Fatou's lemma its average degree is at most 6 , so that Theorem 1.1 implies it is almost surely $\mathrm{CP}$ parabolic. If in addition $(G, \rho)$ has bounded degrees, then it is recurrent by He-Schramm [28]. In particular, this gives an alternative proof of the Benjamini-Schramm Recurrence Theorem [19] in the primary case of a one-ended limit. We handle the remaining cases in Section 4.1. Unlike the proof of [19], whose main ingredient is a quantitative estimate for finite circle packings [19, Lemma 2.3], our method works with infinite triangulations directly and implies the following generalisation:

Proposition 1.2. Any unimodular, simple, one-ended random rooted planar triangulation $(G, \rho)$ with bounded degrees and $\mathbb{E}[\operatorname{deg}(\rho)]=6$ is almost surely recurrent.

This trivially extends the Benjamini-Schramm result, since any local limit of finite planar graphs is unimodular. An important open question is whether every unimodular random graph is a Benjamini-Schramm limit of finite graphs. In a forthcoming paper [7], we show that any unimodular planar graph $G$ is a limit of some sequence of finite graphs $G_{n}$, and that if $G$ is a triangulation with $\mathbb{E}[\operatorname{deg}(\rho)]=6$ then $G_{n}$ can also be taken to be planar. In particular, any graph 
to which Proposition 1.2 applies is also a local limit of finite planar graphs with bounded degrees. Consequently there are no graphs to which this result applies and the Benjamini-Schramm Theorem does not. Note however, that for a given unimodular planar triangulation, it may not be obvious how to find this sequence of graphs. We remark that the dichotomy of Theorem 1.1 has many extensions, applying to more general maps and holding further properties equivalent. We address these in [7]. See [11] and [28, Theorem 10.2] for earlier connections between the CP type and degree distributions in the deterministic setting.

Our method of proof relies on the deep theorem of Schramm [39] that the circle packing of a triangulation in the disc or the plane is unique up to Möbius transformations fixing the disc or the plane as appropriate. We use this fact throughout the paper in an essential way: it implies that any quantity derived from the circle packing in the disc or the plane that is invariant to Möbius transformations is determined by the graph $G$ and not by our choice of circle packing. Key examples of such quantities are angles between adjacent edges in the associated drawings with hyperbolic or Euclidean geodesics (see Section 4), hyperbolic radii of circles in the hyperbolic case, and ratios of Euclidean radii in the parabolic case.

Boundary Theory. Throughout, we realize the hyperbolic plane as the Poincaré disc $\{|z|<1\}$ with metric $d_{\text {hyp }}$. The unit circle $\{|z|=1\}$ is the boundary of the hyperbolic plane in several geometric and probabilistic senses. For a general graph embedded in the hyperbolic plane, the unit circle may or may not coincide with probabilistic notions of the graph's boundary.

When a bounded degree triangulation is circle packed in the disc, Benjamini and Schramm [16] showed that the random walk converges to a point in the circle almost surely and that the law of the limit point has full support and no atoms. More recently, it was shown by the first and third authors together with Barlow and Gurel-Gurevich [4] that the unit circle is a realisation of both the Poisson and Martin boundaries of the triangulation. Similar results regarding square tiling were obtained in [17] and [23].

Again, these theorems fail for some triangulations with unbounded degrees. Starting with any CP hyperbolic triangulation, one can add circles in the interstices of the packing so as to create drifts along arbitrary paths. In this way, one can force the random walk to spiral in the unit disc and not converge to any point in the boundary. One can also create a graph for which the walk can converge to a single boundary point from two or more different angles each with positive probability, so that the exit measure is atomic and the unit circle is no longer a realisation of the Poisson boundary. Our next result recovers the boundary theory in the unimodular setting.

When $\mathcal{C}$ is a circle packing of a graph $G$ in the disc $\mathbb{D}$, we write $\mathcal{C}=(z, r)$ 
where $z(v)$ is the (Euclidean) centre of the circle corresponding to $v$, and $r(v)$ is its Euclidean radius. Recall that the hyperbolic metric on the unit disc is defined by

$$
\left|d_{\text {hyp }}(z)\right|=\frac{2|d z|}{1-|z|^{2}},
$$

and that circles in the Euclidean metric are also hyperbolic circles (with different centres and radii). We write $z_{h}(v)$ and $r_{h}(v)$ for the hyperbolic centre and radius of the circle corresponding to $v$. We use $P_{v}^{G}$ and $E_{v}^{G}$ to denote the probability and expectation (conditioned on $G$ ) with respect to random walk $\left(X_{n}\right)_{n \geq 0}$ on $G$ started from a vertex $v$.

Theorem 1.3. Let $(G, \rho)$ be a simple, one-ended, $C P$ hyperbolic unimodular random planar triangulation with $\mathbb{E}\left[\operatorname{deg}^{2}(\rho)\right]<\infty$. Let $\mathcal{C}$ be a circle packing of $G$ in the unit disc, and let $\left(X_{n}\right)$ be a simple random walk on $G$. The following hold conditional on $(G, \rho)$ almost surely:

1. $z\left(X_{n}\right)$ and $z_{h}\left(X_{n}\right)$ both converge to a (random) point denoted $\Xi \in \partial \mathbb{D}$,

2. The law of $\Xi$ has full support $\partial \mathbb{D}$ and no atoms.

3. $\partial \mathbb{D}$ is a realisation of the Poisson boundary of $G$. That is, for every bounded harmonic function $h$ on $G$ there exists a bounded measurable function $g$ : $\partial \mathbb{D} \rightarrow \mathbb{R}$ such that

$$
h(v)=E_{v}^{G}[g(\Xi)] .
$$

We refer to the law of $\Xi$ conditional on $(G, \rho)$ as the exit measure from $v$. In Section 7 we extend this result to weighted and non-simple triangulations, with the obvious changes. One ingredient in the proof of the absence of atoms is a more general observation, Lemma 5.2, which states roughly that exit measures on boundaries of stationary graphs are either non-atomic or trivial almost surely.

Our final result relates exponential decay of the Euclidean radii along the random walk to speed in the hyperbolic metric.

Theorem 1.4. Let $(G, \rho)$ be a simple, one-ended, $C P$ hyperbolic unimodular random rooted planar triangulation with $\mathbb{E}\left[\operatorname{deg}^{2}(\rho)\right]<\infty$ and let $\mathcal{C}$ be a circle packing of $G$ in the unit disc. Then almost surely

$$
\lim _{n \rightarrow \infty} \frac{d_{h y p}\left(z_{h}(\rho), z_{h}\left(X_{n}\right)\right)}{n}=\lim _{n \rightarrow \infty} \frac{-\log r\left(X_{n}\right)}{n}>0 .
$$

In particular, both limits exist. Moreover, the limits do not depend on the choice of packing, and if $(G, \rho)$ is ergodic then this limit is an almost sure constant.

Thus the random walk $\left(X_{n}\right)$ has positive asymptotic speed in the hyperbolic metric, the Euclidean radii along the walk decay exponentially, and the two rates agree. 
Organization of the paper. In Section 2 we review the motivating examples of unimodular hyperbolic random triangulations to which our results apply. In Section 3.1 and Section 3.2 we give background on unimodularity, reversibility and related topics. In Section 3.3 we recall Aldous and Lyons's notion of invariant amenability [1] and prove one of its important consequences. In Section 3.4 we recall the required results on circle packing and discuss measurability. Section 4 contains the proof of Theorem 1.1 as well as a discussion of how to handle the remaining (easier) cases of the Benjamini-Schramm Theorem. Theorem 1.3 is proved in Section 5 and Theorem 1.4 is proved in Section 6. Background on the Poisson boundary is provided before the proof of Theorem 1.3(3) in Section 5.3. In Section 7 we discuss extensions of our results to non-simple and weighted triangulations. We end with some open problems in Section 8.

\section{Examples}

Benjamini-Schramm limits of random maps have been objects of great interest in recent years, serving as discrete models of 2-dimensional quantum gravity. Roughly, the idea is to consider a uniformly random map from some class of rooted maps (e.g. all triangulations or quadrangulations of the sphere of size $n$ ) and take a local limit as the size of the maps tends to infinity. The first such construction was the UIPT [10]; see also [3, 13, 21, 6, 5].

Curien's PSHT. Recently, hyperbolic versions of the UIPT and related maps have been constructed: half-plane versions in [9] and full-plane versions in [20]. These are constructed directly, and are believed but not yet known to be the limits of finite maps (see below). The full plane triangulations form a one (continuous) parameter family $\left\{T_{\kappa}\right\}_{\kappa \in(0,2 / 27)}$ (known as the PSHT, for Planar Stochastic Hyperbolic Triangulation). They are reversible and ergodic, have anchored expansion and are therefore invariantly non-amenable. The degree of the root in $T_{\kappa}$ is known to have an exponential tail, so that all of its moments are finite. These triangulations are not simple, so our main results do not apply to them directly, but by considering their simple cores we are still able to obtain a geometric representation of their Poisson boundary (see Section 7).

Benjamini-Schramm limits of maps in high genus. It is conjectured that the PSHT $T_{\kappa}$ is the Benjamini-Schramm limit of the uniform triangulation with $n$ vertices of a surface of genus $\lfloor\theta n\rfloor$, for some $\theta=\theta(\kappa)$ (see e.g. [36] for precise definitions of maps on general surfaces). In our upcoming paper [7] we prove that all one-ended unimodular random rooted planar triangulations are also BenjaminiSchramm limits of finite triangulations. If the triangulation has expected degree 
greater than 6 , then the finite approximating triangulations necessarily have genus linear in their size.

In the context of circle packing, it may be particularly interesting to take the Benjamini-Schramm limit $(T, \rho)$ of the uniform simple triangulation with $n$ vertices of the $\lfloor\theta n\rfloor$-holed torus $T_{n}$. This limit (which we conjecture exists) should be a simple variant of the PSHT. Letting $\rho_{n}$ be a uniformly chosen root of $T_{n}$, it should also be the case that $\mathbb{E}\left[\operatorname{deg}\left(\rho_{n}\right)\right] \rightarrow \mathbb{E}[\operatorname{deg}(\rho)]>6$ and $\mathbb{E}\left[\operatorname{deg}(\rho)^{2}\right]<\infty$, so that our results would be applicable to the circle packing of $(T, \rho)$.

Delaunay triangulations of the hyperbolic plane. Start with a Poisson point process in the hyperbolic plane with intensity $\lambda$ times the hyperbolic area measure, and add a root point at the origin. Consider now the Delaunay triangulation with this point process as its vertex set, where three vertices $u, v, w$ form a triangle if the circle through $u, v, w$ contains no other points of the process. This triangulation, known as the Poisson-Delaunay triangulation, is naturally embedded in the hyperbolic plane with hyperbolic geodesic edges. These triangulations, studied in $[18,15]$, are unimodular when rooted at the point at the origin. They are known to have anchored expansion [15] and are therefore invariantly non-amenable. (We also get a new proof of non-amenability from Theorem 1.1, as one can show the expected degree to be greater than six by transporting angles as in the proof of Theorem 1.1.) The Poisson-Delaunay triangulations are also simple and one-ended, and the degree of the root has finite second moment, so that our results apply directly to their circle packings.

\section{Background and Definitions}

\subsection{Unimodular random graphs and maps}

Unimodularity of graphs (both fixed and random) has proven to be a useful and natural property in a number of settings. We give here the required definitions and some of their consequences, and refer the reader to $[1,33]$ for further background.

A rooted graph $(G, \rho)$ is a graph $G=(V, E)$ with a distinguished vertex $\rho$ called the root. We will allow our graphs to contain self-loops and multiple edges, and refer to graphs without either as simple. A graph is said to be one-ended if the removal of any finite set of vertices leaves precisely one infinite connected component. A graph isomorphism between two rooted graphs is a rooted graph isomorphism if it preserves the root.

A map is a proper (that is, with non-intersecting edges) embedding of a connected graph into a surface, viewed up to orientation preserving homeomorphisms of the surface, so that all connected components of the complement (called faces) 
are topological discs. ${ }^{1}$ The map is planar if the surface is homeomorphic to an open subset of the sphere, and is simply connected if the surface is homeomorphic to the sphere or the plane. A map is a triangulation if every face is incident to exactly three edges. Note that an infinite planar triangulation is simply connected if and only if it is one-ended.

Every connected graph $G$ can be made into a metric space by endowing it with the shortest path metric $d_{G}$. By abuse of notation, we use the ball $B_{n}(G, u)$ to refer both to the set of vertices $\left\{v \in V: d_{G}(u, v) \leq n\right\}$ and the induced subgraph on this set, rooted at $u$. The balls in a map inherit a map structure from the full map.

The local topology on the space of rooted connected graphs (introduced in [19]) is the topology induced by the metric

$$
d_{\mathrm{loc}}\left((G, \rho),\left(G^{\prime}, \rho^{\prime}\right)\right)=e^{-R} \quad \text { where } \quad R=\sup \left\{n \geq 0: B_{n}(G, \rho) \cong B_{n}\left(G^{\prime}, \rho^{\prime}\right)\right\} .
$$

The local topology on rooted maps is defined similarly by requiring the isomorphism of the balls to be an isomorphism of rooted maps. We denote by $\mathcal{G}$. and $\mathcal{M}$. the spaces of isomorphism classes of rooted connected graphs and of maps with their respective local topologies. Random rooted graphs and maps are Borel random variables taking values in these spaces.

Several variants of these spaces will also be of use. A (countably) marked graph is a graph together with a mark function $m: V \cup E \rightarrow M$ which gives every edge and vertex a mark in some countable set $M$. A graph isomorphism between marked graphs is an isomorphism of marked graphs if it preserves the marks. The local topologies on rooted marked graphs and maps is defined in the obvious way. These spaces are denoted $\mathcal{G}_{\bullet}^{M}$ and $\mathcal{M}_{\bullet}^{M}$. Sometimes we will consider maps with marks only on vertices or only on edges; these fit easily into our framework. Marked graphs are special cases of what Aldous and Lyons [1] call networks, for which the marks may take values in any separable complete metric space.

Similarly, we define $\mathcal{G}_{\bullet \bullet}$ (resp. $\mathcal{M}_{\bullet \bullet}$ ) to be the spaces of doubly rooted (that is, with a distinguished ordered pair of vertices) connected graphs (resp. maps) $(G, u, v)$. These spaces, along with their marked versions, are equipped with natural variants of the local topology. All such spaces we consider are Polish.

A mass transport is a non-negative Borel function $f: \mathcal{G}_{\bullet \bullet} \rightarrow \mathbb{R}_{+}$. A random rooted graph $(G, \rho)$ is said to be unimodular if it satisfies the mass transport principle: for any mass transport $f$,

$$
\mathbb{E}\left[\sum_{v \in V(G)} f(G, \rho, v)\right]=\mathbb{E}\left[\sum_{v \in V(G)} f(G, v, \rho)\right]
$$

\footnotetext{
${ }^{1}$ There is an additional constraint regarding boundaries of faces of infinite degree. However, this condition is automatically satisfied for triangulations and for simply connected maps, so that we need not worry about it in this paper.
} 
In other words,

'Expected mass out equals expected mass in.'

This definition generalises naturally to define unimodular marked graphs and maps. Importantly, any finite graph $G$ with a uniformly chosen root vertex $\rho$ satisfies the mass transport principle.

The laws of unimodular random rooted graphs form a weakly closed, convex subset of the space of probability measures on $\mathcal{G}_{\bullet}$, so that weak limits of unimodular random graphs are unimodular. In particular, a weak limit of finite graphs with uniformly chosen roots is unimodular: such a limit of finite graphs is referred to as a Benjamini-Schramm limit. It is a major open problem to determine whether all unimodular random rooted graphs arise as Benjamini-Schramm limits of finite graphs $[1, \S 10]$. As mentioned in Section 2, we provide a positive solution to this problem in the planar case in the upcoming work [7], proving that every simply connected unimodular random rooted planar map is a Benjamini-Schramm limit of finite maps.

A common use of the mass transport principle to obtain proofs by contradiction is the following. If $(G, \rho)$ is a unimodular random rooted graph and $f$ is a mass transport such that the mass sent out from each vertex $\sum_{v} f(G, u, v) \leq M$ is uniformly bounded almost surely, then almost surely there are no vertices that receive infinite mass: if vertices receiving infinite mass were to exist with positive probability, the root would be such a vertex with positive probability [1, Lemma 2.3], contradicting the mass transport principle.

\subsection{Random walk, reversibility and ergodicity}

Recall that the simple random walk on a graph is the Markov chain that chooses $X_{n+1}$ from among the neighbours of $X_{n}$ weighted by the number of shared edges. Define $\mathcal{G}_{\leftrightarrow}\left(\right.$ resp. $\left.\mathcal{M}_{\leftrightarrow}\right)$ to be spaces of isomorphism classes of graphs (resp. maps) equipped with a bi-infinite path $\left(G,\left(x_{n}\right)_{n \in \mathbb{Z}}\right)$, which we endow with a natural variant of the local topology. When $(G, \rho)$ is a random graph or map, we let $\left(X_{n}\right)_{n \geq 0}$ and $\left(X_{-n}\right)_{n \geq 0}$ be two independent simple random walks started from $\rho$ and consider $\left(G,\left(X_{n}\right)_{n \in \mathbb{Z}}\right)$ to be a random element of $\mathcal{G}_{\leftrightarrow}$ or $\mathcal{M}_{\leftrightarrow}$ as appropriate.

A random rooted graph $(G, \rho)$ is stationary if $(G, \rho) \stackrel{d}{=}\left(G, X_{1}\right)$ and reversible if $\left(G, \rho, X_{1}\right) \stackrel{d}{=}\left(G, X_{1}, \rho\right)$ as doubly rooted graphs. Equivalently, $(G, \rho)$ is reversible if and only if $\left(G,\left(X_{n}\right)_{n \in \mathbb{Z}}\right)$ is stationary with respect to the shift:

$$
\left(G,\left(X_{n}\right)_{n \in \mathbb{Z}}\right) \stackrel{d}{=}\left(G,\left(X_{n+k}\right)_{n \in \mathbb{Z}}\right) \text { for every } k \in \mathbb{Z} .
$$

To see this, it suffices to prove that if $(G, \rho)$ is a reversible random graph then $\left(X_{1}, \rho, X_{-1}, X_{-2}, \ldots\right)$ has the law of a simple random walk started from $X_{1}$. But 
$\left(\rho, X_{-1}, \ldots\right)$ is a simple random walk started from $\rho$ independent of $X_{1}$ and, conditional on $\left(G, X_{1}\right)$, reversibility implies that $\rho$ is uniformly distributed among the neighbours of $X_{1}$, so $\left(X_{1}, \rho, X_{-1}, X_{-2}, \ldots\right)$ has the law of a simple random walk as desired.

We remark that if $(G, \rho)$ is stationary but not necessarily reversible, it is still possible to extend the walk to a doubly infinite path $\left(X_{n}\right)_{n \in \mathbb{Z}}$ so that $G$ is stationary along the path. The difference is that in the reversible case the past $\left(X_{n}\right)_{n \leq 0}$ is itself a simple random walk with the same law as the future.

Reversibility is related to unimodularity via the following bijection, which is implicit in [1] and proven explicitly in [12]: if $(G, \rho)$ is reversible, then biasing by $\operatorname{deg}(\rho)^{-1}$ (i.e. reweighing the law of $(G, \rho)$ by the Radon-Nikodym derivative $\left.\operatorname{deg}(\rho)^{-1} / \mathbb{E}\left[\operatorname{deg}(\rho)^{-1}\right]\right)$ gives an equivalent unimodular random rooted graph, and conversely if $(G, \rho)$ is a unimodular random rooted graph with finite expected degree, then biasing by $\operatorname{deg}(\rho)$ gives an equivalent reversible random rooted graph. Thus, the laws of reversible random rooted graphs are in bijection with the laws of unimodular random rooted graphs for which the root degree has finite expectation.

An event $A \subset \mathcal{G}_{\leftrightarrow}$ is said to be invariant if $\left(G,\left(X_{n}\right)_{n \in \mathbb{Z}}\right) \in A$ implies $\left(G,\left(X_{n+k}\right)_{n \in \mathbb{Z}}\right) \in A$ for each $k \in \mathbb{Z}$. A reversible or unimodular random graph is said to be ergodic if the law of $\left(G,\left(X_{n}\right)_{n \in \mathbb{Z}}\right)$ gives each invariant event probability either zero or one. An event $A \subseteq \mathcal{G}$ • is rerooting-invariant if $(G, \rho) \in A$ implies $(G, v) \in A$ for every vertex $v$ of $G$.

Theorem 3.1 (Characterisation of ergodicity $[1, \S 4])$. Let $(G, \rho)$ be a unimodular random rooted graph with $\mathbb{E}[\operatorname{deg}(\rho)]<\infty$ (resp. a reversible random rooted graph). The following are equivalent.

1. $(G, \rho)$ is ergodic.

2. Every rerooting-invariant event $A \subseteq \mathcal{G}$. has probability in $\{0,1\}$.

3. The law of $(G, \rho)$ is an extreme point of the weakly closed convex set of laws of unimodular (resp. reversible) random rooted graphs.

(The equivalence of items 2 and 3 holds for unimodular random rooted graphs without the assumption of finite expected degree.) A consequence of the extremal characterisation is that every unimodular random rooted graph is a mixture of ergodic unimodular random rooted graphs, meaning that it may be sampled by first sampling a random law of an ergodic unimodular random rooted graph, and then sampling from this randomly chosen law - this is known as an ergodic decomposition and its existence is a consequence of Choquet's Theorem. In particular, whenever we want to prove that a unimodular random rooted graph with some almost sure property also has some other almost sure property, it suffices to consider the ergodic case. The same comment applies for reversible random rooted graphs. 


\subsection{Invariant amenability}

We begin with a brief review of general amenability, before combining it with unimodularity for the notion of invariant amenability. We refer the reader to $[33, \S 6]$ for further details on amenability in general, and $[1, \S 8]$ for invariant amenability.

A weighted graph is a graph together with a weight function $w: E \rightarrow \mathbb{R}_{+}$. Unweighted multigraphs may always be considered as weighted graphs by setting $w \equiv 1$. The weight function is extended to vertices by $w(x)=\sum_{e \ni x} w(e)$, and (with a slight abuse of notation) to sets of edges or vertices by additivity. The simple random walk $X=\left(X_{n}\right)_{n \geq 0}$ on a weighted graph is the Markov chain on $V$ with transition probabilities $p(x, y)=w(x, y) / w(x)$. Here, our graphs are allowed to have infinite degree provided $w(v)$ is finite for every vertex.

The (edge) Cheeger constant of an infinite weighted graph is defined to be

$$
\mathbf{i}_{E}(G)=\inf \left\{\frac{w\left(\partial_{E} W\right)}{w(W)}: \emptyset \neq W \subset V \text { finite }\right\}
$$

where $\partial_{E} W$ denotes the set of edges with exactly one end in $W$. A graph is said to be amenable if its Cheeger constant is zero and non-amenable if it is positive.

The Markov operator associated to simple random walk on $G$ is the bounded, self-adjoint operator from $L^{2}(V, w)$ to itself defined by $(P f)(u)=\sum p(u, v) f(v)$. The norm of this operator is commonly known as the spectral radius of the graph. If $u, v \in V$ then the transition probabilities are given by $p_{n}(u, v)=$ $\left\langle P^{n} \mathbb{1}_{v}, \mathbb{1}_{u} / w(u)\right\rangle_{w}$, so that, by Cauchy-Schwarz,

$$
p_{n}(u, v) \leq \sqrt{\frac{w(v)}{w(u)}}\|P\|_{w}^{n}
$$

and in fact $\|P\|_{w}=\limsup _{n \rightarrow \infty} p_{n}(u, v)^{1 / n}$. A fundamental result, originally proved for Cayley graphs by Kesten [30], is that the spectral radius of a weighted graph is less than one if and only if the graph is non-amenable (see [33, Theorem 6.7] for a modern account). As an immediate consequence, non-amenable graphs are transient for simple random walk.

\subsubsection{Invariant amenability}

There are natural notions of amenability and expansion for unimodular random networks due to Aldous and Lyons [1]. A percolation on a unimodular random rooted graph $(G, \rho)$ is a random assignment of $\omega: E \cup V \rightarrow\{0,1\}$ such that the marked graph $(G, \rho, \omega)$ is unimodular. We think of $\omega$ as a random subgraph of $G$ consisting of the 'open' edges and vertices $\omega(e)=1, \omega(v)=1$, and may assume without loss of generality that if an edge is open then so are both of its endpoints. 
The cluster $K_{\omega}(v)$ at a vertex $v$ is the connected component of $v$ in $\omega$, i.e. the set of vertices for which there is a path of open edges to $v$ (by convention, if $\omega(v)=0$ or if there are no open edges touching $v$ we put $K_{\omega}(v)=\{v\}$ ). A percolation is said to be finitary if all of its clusters are finite almost surely. The invariant Cheeger constant of an ergodic unimodular random rooted graph $(G, \rho)$ is defined to be

$$
\mathrm{i}^{\text {inv }}((G, \rho))=\inf \left\{\mathbb{E}\left[\frac{\left|\partial_{E} K_{\omega}(\rho)\right|}{\left|K_{\omega}(\rho)\right|}\right]: \omega \text { a finitary percolation on }(G, \rho)\right\} .
$$

The invariant Cheeger constant is closely related to another quantity: mean degrees in finitary percolations. Let $\operatorname{deg}_{\omega}(\rho)$ denote the degree of $\rho$ in $\omega$ (seen as a subgraph; if $\rho \notin \omega$ we set $\left.\operatorname{deg}_{\omega}(\rho)=0\right)$ and let

$$
\alpha((G, \rho))=\sup \left\{\mathbb{E}\left[\operatorname{deg}_{\omega}(\rho)\right]: \omega \text { a finitary percolation on }(G, \rho)\right\} .
$$

An easy application of the mass transport principle [1, Lemma 8.2] shows that, for any finitary percolation $\omega$,

$$
\mathbb{E}\left[\operatorname{deg}_{\omega}(\rho)\right]=\mathbb{E}\left[\frac{\sum_{v \in K_{\omega}(\rho)} \operatorname{deg}_{\omega}(v)}{\left|K_{\omega}(\rho)\right|}\right] .
$$

It follows that

$$
\mathbb{E}[\operatorname{deg}(\rho)]=\mathrm{i}^{\mathrm{inv}}((G, \rho))+\alpha((G, \rho))
$$

so that if $\mathbb{E}[\operatorname{deg}(\rho)]<\infty$ then $\mathbf{i}^{\text {inv }}((G, \rho))$ is positive if and only if $\alpha((G, \rho))$ is strictly smaller than $\mathbb{E}[\operatorname{deg}(\rho)]$.

We say that an ergodic unimodular random rooted graph $(G, \rho)$ is invariantly amenable if $\mathbf{i}^{\text {inv }}((G, \rho))=0$ and invariantly non-amenable otherwise. Note that this is a property of the law of $(G, \rho)$ and not of an individual graph. We remark that what we are calling invariant amenability was called amenability when it was introduced by Aldous and Lyons $[1, \S 8]$. We qualify it as invariant to distinguish it from the more classical notion, which we also use below. While any invariantly amenable graph is trivially amenable, the converse is generally false. An example is a 3-regular tree where each edge is replaced by a path of independent length with unbounded distribution; see [1] for a more detailed discussion.

An important property of invariantly non-amenable graphs was first proved for Cayley graphs by Benjamini, Lyons and Schramm [14]. Aldous and Lyons [1] noted that the proof carried through with minor modifications to the case of invariantly non-amenable unimodular random rooted graphs, but did not provide a proof. As this property is crucial to our arguments, we provide a proof for completeness, which the reader may wish to skip. When $(G, \rho)$ is an ergodic unimodular random rooted graph, we say that a percolation $\omega$ on $G$ is ergodic if $(G, \rho, \omega)$ is ergodic as a unimodular random rooted marked graph. The following is stated slightly differently from both Theorem 3.2 in [14] and Theorem 8.13 in [1]. 
Theorem 3.2. Let $(G, \rho)$ be an invariantly non-amenable ergodic unimodular random rooted graph with $\mathbb{E}[\operatorname{deg}(\rho)]<\infty$. Then $G$ admits an ergodic percolation $\omega$ so that $\mathbf{i}_{E}(\omega)>0$ and vertices in $\omega$ have uniformly bounded degrees in $G$.

Let us stress that the condition of uniformly bounded degrees is for the degrees in the full graph $G$, and not the degrees in the percolation.

Remark 3.3. This theorem plays the same role for invariant non-amenability as Virág's oceans and islands construction [43] does for anchored expansion [43, 8, 15]. In particular, it gives us a percolation $\omega$ such that the induced network $\bar{\omega}$ is non-amenable (see the proof of Lemma 5.1).

Proof. Let $\omega_{0}$ be the percolation induced by vertices of $G$ of degree at most $M$ and the edges connecting any two such vertices. By monotone convergence, and since $\alpha((G, \rho))<\mathbb{E} \operatorname{deg}(\rho)$, we can take $M$ to be large enough that $\mathbb{E} \operatorname{deg}_{\omega_{0}}(\rho)>$ $\alpha((G, \rho))$. This gives a percolation with bounded degrees. We shall modify it further to get non-amenability as follows. Fix $\delta>0$ by

$$
3 \delta=\mathbb{E}\left[\operatorname{deg}_{\omega_{0}}(\rho)\right]-\alpha((G, \rho)) .
$$

Construct inductively a decreasing sequence of site percolations $\omega_{n}$ as follows. Given $\omega_{n}$, let $\eta_{n}$ be independent Bernoulli(1/2) site percolations on $\omega_{n}$, and for each set of vertices $W$ let $\partial_{E}^{\omega_{n}} W$ denote the set of edges of $\omega_{n}$ in the boundary of $W$. If $K$ is a finite connected cluster of $\eta_{n}$, with small boundary in $\omega_{n}$, we remove it to construct $\omega_{n+1}$. More precisely, let $\omega_{n+1}=\omega_{n} \backslash \gamma_{n}$, where $\gamma_{n}$ is the subgraph of $\omega_{n}$ induced by the vertex set

$$
\bigcup\left\{K: K \text { a finite cluster in } \eta_{n} \text { with }\left|\partial_{E}^{\omega_{n}}(K)\right|<\delta|K|\right\} .
$$

Let $\omega=\cap \omega_{n}$ be the limit percolation, which is clearly ergodic. We shall show below that $\omega \neq \emptyset$. Any finite connected set in $\omega$ appears as a connected cluster in $\eta_{n}$ for infinitely many $n$. If such a set $S$ has $\left|\partial_{E}^{\omega} S\right|<\delta|S|$ then it would have been removed at some step, and so $\omega$ has $\left|\partial_{E}^{\omega} S\right| \geq \delta|S|$ for all finite connected $S$. Since degrees are bounded by $M$, this implies $\mathbf{i}_{E}(\omega) \geq \delta / M>0$.

It remains to show that $\omega \neq \emptyset$. For some $n$, and any vertex $u$, let $K(u)$ be its cluster in $\eta_{n}$. Consider the mass transport

$$
f_{n}(u, v)= \begin{cases}\operatorname{deg}_{\omega_{n}}(v) /|K(u)| & u \in \gamma_{n} \text { and } v \in K(u), \\ E(v, K(u)) /|K(u)| & u \in \gamma_{n} \text { and } v \in \omega_{n} \backslash K(u), \\ 0 & u \notin \gamma_{n} .\end{cases}
$$

Here $E(v, K(u))$ is the number of edges between $v$ and $K(u)$. We have that the total mass into $v$ is the difference $\operatorname{deg}_{\omega_{n}}(v)-\operatorname{deg}_{\omega_{n+1}}(v)$ (where the degree is 0 for 
vertices not in the percolation) while the mass sent from a vertex $v \in \gamma_{n}$ is twice the number of edges with either end in $K(v)$, divided by $|K(v)|$. Applying the mass transport principle we get

$$
\mathbb{E}\left[\operatorname{deg}_{\omega_{n}}(\rho)-\operatorname{deg}_{\omega_{n+1}}(\rho)\right]=\mathbb{E}\left[\frac{\sum_{v \in K(\rho)} \operatorname{deg}_{\gamma_{n}}(v)+2\left|\partial_{E}^{\omega_{n}}(K(\rho))\right|}{|K(\rho)|} \mathbb{1}_{\rho \in \gamma_{n}}\right]
$$

By a second transport, of $\operatorname{deg}_{\gamma_{n}}(u) /|K(u)|$ from every $u \in \gamma_{n}$ to each $v \in K(u)$, we see that

$$
\mathbb{E}\left[\frac{\sum_{v \in K(\rho)} \operatorname{deg}_{\gamma_{n}}(v)}{|K(\rho)|} \mathbb{1}_{\rho \in \gamma_{n}}\right]=\mathbb{E}\left[\operatorname{deg}_{\gamma_{n}}(\rho)\right] .
$$

Additionally, on the event $\left\{\rho \in \gamma_{n}\right\}$, we have by definition that

$$
\left|\partial_{E}^{\omega_{n}}(K(\rho))\right| /|K(\rho)| \leq \delta
$$

Plugging these two in (3.3) gives

$$
\mathbb{E}\left[\operatorname{deg}_{\omega_{n}}(\rho)-\operatorname{deg}_{\omega_{n+1}}(\rho)\right] \leq \mathbb{E}\left[\operatorname{deg}_{\gamma_{n}}(\rho)\right]+2 \delta \mathbb{P}\left(\rho \in \gamma_{n}\right)
$$

Let $\gamma=\cup_{n \geq 1} \gamma_{n}$, which is a percolation since it is defined as a measurable, automorphism invariant function of $(G, \rho)$ and the i.i.d. sequence of Bernoulli percolations $\left(\eta_{n}\right)$. Note the percolations $\gamma_{n}$ are disjoint, so that the event $\rho \in \gamma_{n}$ can occur for at most one $n$ and that $\gamma$ is a finitary percolation. Thus $\mathbb{E}\left[\operatorname{deg}_{\gamma}(\rho)\right]=$ $\sum_{n} \mathbb{E}\left[\operatorname{deg}_{\gamma_{n}}(\rho)\right] \leq \alpha((G, \rho))$. Also, $\sum_{n} \mathbb{P}\left(\rho \in \gamma_{n}\right) \leq 1$. Summing over $n$ gives

$$
\mathbb{E}\left[\operatorname{deg}_{\omega_{0}}(\rho)-\operatorname{deg}_{\omega}(\rho)\right] \leq \alpha((G, \rho))+2 \delta .
$$

The definition of $\delta$ leaves $\mathbb{E}\left[\operatorname{deg}_{\omega}(\rho)\right] \geq \delta$. Thus $\omega$ is indeed non-empty as claimed, completing the proof.

\subsection{Circle packings and vertex extremal length}

Recall that a circle packing $\mathcal{C}$ is a collection of discs of disjoint interior in the plane $\mathbb{C}$. Given a circle packing $\mathcal{C}$, we define its tangency map as the map whose embedded vertex set $V$ corresponds to the centres of circles in $\mathcal{C}$ and whose edges are given by straight lines between the centres of tangent circles. If $\mathcal{C}$ is a packing whose tangency map is isomorphic to $G$, we call $\mathcal{C}$ a packing of $G$.

Theorem 3.4 (Koebe-Andreev-Thurston Circle Packing Theorem [31, 42]). Every finite simple planar map arises as the tangency map of a circle packing. If the map is a triangulation, the packing is unique up to Möbius transformations of the sphere. 
The carrier of a circle packing is the union of all the discs in the packing together with the curved triangular regions enclosed between each triplet of circles corresponding to a face (the interstices). Given some planar domain $D$, we say that a circle packing is in $D$ if its carrier is $D$.

Theorem 3.5 (Rigidity for Infinite Packings, Schramm [39]). Let $G$ be a triangulation, circle packed in either $\mathbb{C}$ or $\mathbb{D}$. Then the packing is unique up to Möbius transformations preserving of $\mathbb{C}$ or $\mathbb{D}$ respectively.

It is often fruitful to think of packings in $\mathbb{D}$ as being circle packings in (the Poincare disc model of) the hyperbolic plane. The uniqueness of the packing in $\mathbb{D}$ up to Möbius transformations may then be stated as uniqueness of the packing in the hyperbolic plane up to isometries of the hyperbolic plane.

The vertex extremal length, defined in [28], from a vertex to infinity on an infinite graph $G$ is defined to be

$$
\operatorname{VEL}_{G}(v, \infty)=\sup _{m} \frac{\inf _{\gamma: v \rightarrow \infty} m(\gamma)^{2}}{\|m\|^{2}},
$$

where the supremum is over measures $m$ on $V(G)$ such that $\|m\|^{2}=\sum m(u)^{2}<\infty$, and the infimum is over paths from $v$ to $\infty$ in $G$. A connected graph is said to be VEL parabolic if $\operatorname{VEL}(v \rightarrow \infty)=\infty$ for some vertex $v$ (and hence for any vertex) and VEL hyperbolic otherwise. The VEL type is monotone in the sense that subgraphs of VEL parabolic graphs are also VEL parabolic. A simple random walk on any VEL hyperbolic graph is transient. For graphs with bounded degrees the converse also holds: Transient graphs with bounded degrees are VEL hyperbolic [28].

Theorem 3.6 (He-Schramm $[27,28])$. Let $G$ be a one-ended, infinite, simple planar triangulation. Then $G$ may be circle packed in either the plane $\mathbb{C}$ or the unit disc $\mathbb{D}$, according to whether it is VEL parabolic or hyperbolic respectively.

The final classical fact about circle packing we will need is the following quantitative version, due to Hansen [25], of the Ring Lemma of Rodin and Sullivan [37], which will allow us to control the radii along a random walk.

Theorem 3.7 (The Sharp Ring Lemma [25]). Let $u$ and $v$ be two adjacent vertices in a circle packed triangulation, and $r(u), r(v)$ the radii of the corresponding circles. There exists a universal positive constant $C$ such that

$$
\frac{r(v)}{r(u)} \leq e^{C \operatorname{deg}(v)} .
$$




\subsubsection{Measurability of Circle Packing}

At several points throughout the paper, we will want to define mass transports in terms of circle packings. In order for these to be measurable functions of the graph, we require measurability of the circle packing. Let $(G, u, v)$ be a doubly rooted triangulation and let $\mathcal{C}(G, u, v)$ be the unique circle packing of $G$ in $\mathbb{D}$ or $\mathbb{C}$ such that the circle corresponding to $u$ is centred at 0 , the circle corresponding to $v$ is centered on the positive real line and, in the parabolic case, the root circle has radius one.

Let $G_{k}$ be an exhaustion of $G$ by finite induced subgraphs with no cut-vertices and such that the complements $G \backslash G_{k}$ are connected. Such an exhaustion exists by the assumption that $G$ is a one-ended triangulation. Form a finite triangulation $G_{k}^{*}$ by adding an extra vertex $\partial_{k}$ and an edge from $\partial_{k}$ to each boundary vertex of $G_{k}$.

Consider first the case when $G$ is CP hyperbolic. By applying a Möbius transformation to some circle packing of $G_{k}^{*}$, we find a unique circle packing $\mathcal{C}_{k}^{*}$ of $G_{k}^{*}$ in $\mathbb{C}^{\infty}$ such that the circle corresponding to $u$ is centred at the origin, the circle corresponding to $v$ is centred on the positive real line and $\partial_{k}$ corresponds to the unit circle $\partial \mathbb{D}$. In the course of the proof of the He-Schramm Theorem, it is shown that this sequence of packings converges to the unique packing of $G$ in $\mathbb{D}$, normalised so that the circle corresponding to $u$ is centred at 0 and the circle corresponding to $v$ is centred on the positive real line.

As a consequence, the centres and radii of the circles of $\mathcal{C}(G, u, v)$ are limits as $r \rightarrow \infty$ of the centre and radius of a graph determined by the ball of radius $r$ around $u$. In particular, they are pointwise limits of continuous functions (with respect to the local topology on graphs) and hence are measurable.

The hyperbolic radii are particularly nice to consider here. Since the circle packing in $\mathbb{D}$ is unique up to isometries of the hyperbolic plane (Möbius maps), the hyperbolic radii do not depend on the choice of packing, and we find that $r_{h}(v)$ is a function of $(G, v)$.

In the CP parabolic case, the same argument works except that the packing $\mathcal{C}_{k}^{*}$ of $G_{k}^{*}$ must be chosen to map $u$ to the unit circle and $\partial_{k}$ to a larger circle also centred at 0 .

\section{Characterisation of the CP type}

Proof of Theorem 1.1. Since $(G, \rho)$ is ergodic, and since the CP type does not depend on the choice of root, the CP type of $G$ is not random. We first relate the circle packing type to the average degree. Suppose $(G, \rho)$ is CP hyperbolic and consider a circle packing of $G$ in the unit disc. Embed $G$ in $\mathbb{D}$ by drawing the hyperbolic geodesics between the hyperbolic centres of the circles in its packing, 

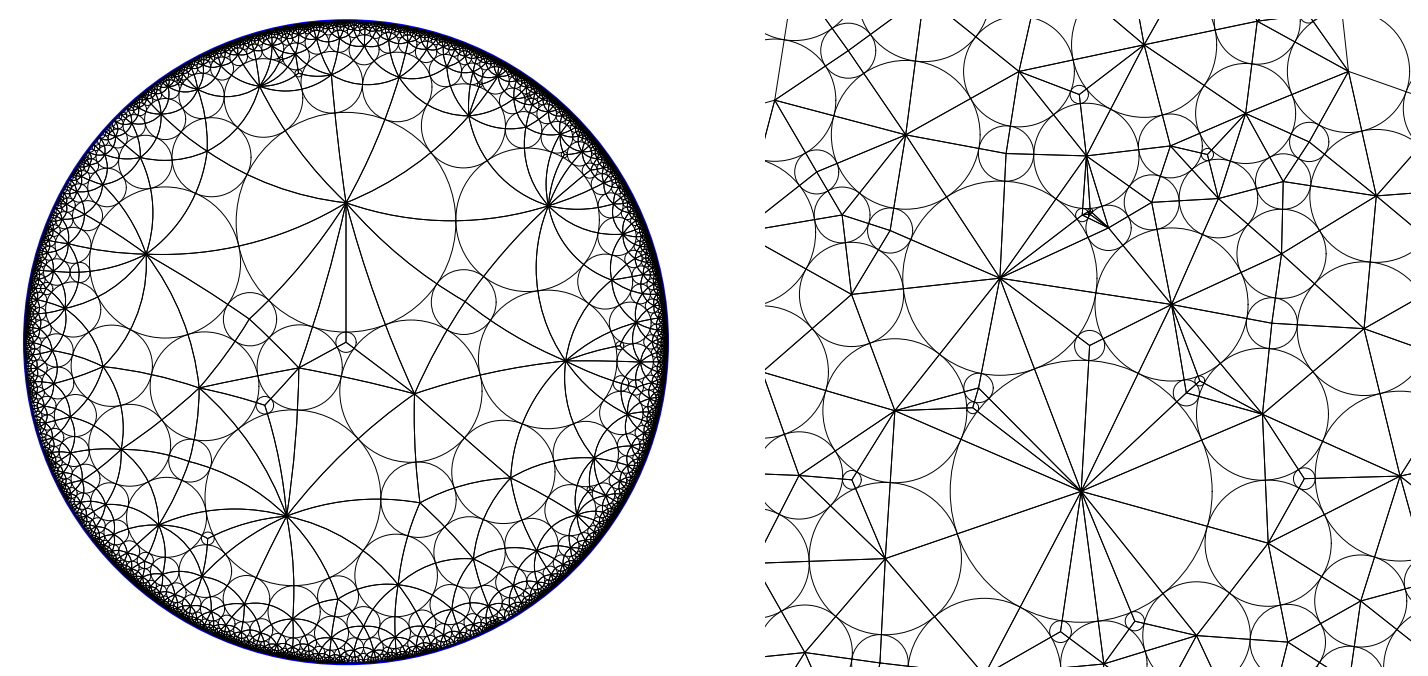

Figure 2: Circle packing induces an embedding of a triangulation with either hyperbolic or Euclidean geodesics, depending on CP type. By rigidity (Theorem 3.5), the angles between pairs of adjacent edges do not depend on the choice of packing.

so that each triangle of $G$ is represented by a hyperbolic triangle (see Figure 2). It is easy to see that this is a proper embedding of $G$. By rigidity of the circle packing (Theorem 3.5), this drawing is determined by the isomorphism class of $G$, up to isometries of the hyperbolic plane.

Define a mass transport as follows. For each face $(u, v, w)$ of the triangulation with angle $\beta$ at $u$, transport $\beta$ from $u$ to each of $u, v, w$. If $u$ and $v$ are adjacent, the transport from $u$ to $v$ has contributions from both faces containing the edge, and the transport from $u$ to itself has a term for each face containing $u$. By rigidity (Theorem 3.5), these angles are independent of the choice of circle packing, so that the mass sent from $u$ to $v$ is a measurable function of $(G, u, v)$.

For each face $f$ of $G$, let $\theta(f)$ denote the sum of the internal angles in $f$ in the drawing. The sum of the angles of a hyperbolic triangle is $\pi$ minus its area, so $\theta(f)<\pi$ for each face $f$. Each vertex $u$ sends each angle 3 times, for a total mass out of exactly $6 \pi$. A vertex receives mass

$$
\sum_{f: u \in f} \theta(f)<\pi \operatorname{deg}(u)
$$

Applying the mass transport principle,

$$
6 \pi<\pi \mathbb{E}[\operatorname{deg}(\rho)] .
$$

Thus if $G$ is CP hyperbolic then $\mathbb{E}[\operatorname{deg}(\rho)]>6$. 
In the $\mathrm{CP}$ parabolic case, we may embed $G$ in $\mathbb{C}$ by drawing straight lines between the centres of the circles in its packing in the plane. By rigidity, this embedding is determined up to translation and scaling, and in particular all angles are determined by $G$. Since the sum of angles in a Euclidean triangle is $\pi$, the same transport as above applied in the CP parabolic case shows that $\mathbb{E}[\operatorname{deg}(\rho)]=6$.

We now turn to amenability. Euler's formula implies that the average degree of any finite simple planar graph is at most 6 . It follows that

$$
\alpha((G, \rho))=\sup \left\{\mathbb{E}\left[\frac{\sum_{v \in K_{\omega}(\rho)} \operatorname{deg}_{\omega}(v)}{\left|K_{\omega}(\rho)\right|}\right]: \omega \text { a finitary percolation }\right\} \leq 6 .
$$

If $G$ is CP hyperbolic then $\mathbb{E}[\operatorname{deg}(\rho)]>6$, so that $\alpha((G, \rho))<\mathbb{E}[\operatorname{deg}(\rho)]$ and $(G, \rho)$ is invariantly non-amenable.

Conversely, suppose $G$ is invariantly non-amenable. By Theorem 3.2, $G$ almost surely admits a percolation $\omega$ which has positive Cheeger constant and bounded degrees. Such an $\omega$ is transient and since it has bounded degree it is also VEL hyperbolic. By monotonicty of the vertex extremal length, $G$ is almost surely VEL hyperbolic as well. The He-Schramm Theorem then implies that $G$ is almost surely CP hyperbolic.

Remark 4.1. In the hyperbolic case, let Area $(u)$ be the total area of the triangles surrounding $u$ in its drawing. Since the angle sum in a hyperbolic triangle is $\pi$ minus its area, the mass transport that gives average degree greater than 6 in the hyperbolic case also gives

$$
\mathbb{E}[\operatorname{deg}(\rho)]=6+\frac{1}{\pi} \mathbb{E}[\operatorname{Area}(\rho)],
$$

which relates the expected degree to the density of the circle packing.

\subsection{Completing the proof of the Benjamini-Schramm The- orem}

In this section we complete our new proof of the following theorem of Benjamini and Schramm.

Theorem $4.2([19])$. Let $(G, \rho)$ be a weak local limit of finite planar graphs $G_{n}$ and suppose that $G$ has bounded degrees almost surely. Then $(G, \rho)$ is almost surely recurrent.

Recall that the number of ends of a graph $G$ is the supremum over finite sets $K$ of the number of infinite connected components of $G \backslash K$. As explained in [19], 
it suffices to prove Theorem 4.2 when the graphs $G_{n}$ are simple triangulations. In this case Proposition 1.2 implies a special case of the Benjamini-Schramm Theorem: If $(G, \rho)$ is a simple one-ended triangulation that is a Benjamini-Schramm limit of finite planar triangulations of uniformly bounded degree, then $(G, \rho)$ is recurrent almost surely. At the time, this was the most difficult case.

Thus, to complete the proof of Theorem 4.2 we need to consider the case in which the limit $(G, \rho)$ has multiple ends. We describe below two different methods to handle this case.

Method 1. This proof considers separately three cases, depending on the number of ends of $G$. First, by combining Proposition 6.10 and Theorem 8.13 of [1], we have the following.

Proposition $4.3([1])$. Let $(G, \rho)$ be an ergodic unimodular random rooted graph. Then $G$ has one, two or infinitely many ends almost surely. If $(G, \rho)$ has infinitely many ends almost surely, it is invariantly non-amenable.

We rule out the case of infinitely many ends by showing that local limits of finite planar graphs are invariantly amenable. Recall the celebrated Lipton-Tarjan Planar Separator Theorem [32, Theorem 2] (which can also be proved using circle packing theory [34]).

Theorem 4.4 ([32]). There exists a universal constant $C$ such that for every $m$ and every finite planar graph $G$, there exists a set $S \subset V(G)$ of size at most $C m^{-1 / 2}|G|$ such that every connected component of $G \backslash S$ contains at most $m$ vertices.

Corollary 4.5. Let $(G, \rho)$ be the local limit of a sequence of finite planar maps $G_{n}$ and suppose $\mathbb{E}[\operatorname{deg}(\rho)]<\infty$. Then $(G, \rho)$ is invariantly amenable and hence has at most two ends.

Proof. Let $\omega_{n}^{m}$ be a subset of $V\left(G_{n}\right)$ such that $G_{n} \backslash \omega_{n}^{m}$ has size at most $C m^{-1 / 2}\left|G_{n}\right|$ and every connected component of $\omega_{n}^{m}$ has size at most $m$. The sequence $\left(G_{n}, \rho_{n}, \omega_{n}^{m}\right)$ is tight and therefore has a subsequence converging to $\left(G, \rho, \omega^{m}\right)$ for some finitary percolation $\omega^{m}$ on $(G, \rho)$. Since it is a limit of percolations on finite graphs with a uniform root, the limit is unimodular.

We have that

$$
\mathbb{P}\left(\rho \in \omega^{m}\right) \geq 1-C m^{-1 / 2} \underset{m \rightarrow \infty}{\longrightarrow} 1 .
$$

Similarly, $\mathbb{P}\left(X_{1} \in \omega^{m}\right) \rightarrow 1$. By integrability of $\operatorname{deg}(\rho)$, we have that

$$
\mathbb{E}\left[\operatorname{deg}_{\omega^{m}}(\rho)\right]=\mathbb{E}\left[\mathbb{1}\left(\rho, X_{1} \in \omega^{m}\right) \operatorname{deg}(\rho)\right] \rightarrow \mathbb{E}[\operatorname{deg}(\rho)] .
$$

Thus $\alpha((G, \rho))=\mathbb{E}[\operatorname{deg}(\rho)]$ and hence $(G, \rho)$ is invariantly amenable. 
Finally, we deal with the two-ended case.

Proposition 4.6. Let $(G, \rho)$ be a unimodular random rooted graph with exactly two ends almost surely and suppose $\mathbb{E}[\operatorname{deg}(\rho)]<\infty$. Then $G$ is recurrent almost surely.

Proof. We prove the equivalent statement for $(G, \rho)$ reversible. We may also assume that $(G, \rho)$ is ergodic. Say that a finite set $S$ disconnects $G$ if $G \backslash S$ has two infinite components. Since $G$ is two-ended almost surely, such a set $S$ exists and each infinite component of $G \backslash S$ is necessarily one-ended. We call these two components $G_{1}$ and $G_{2}$. Suppose for contradiction that $G$ is transient almost surely. In this case, a simple random walk $X_{n}$ eventually stays in one of the $G_{i}$, and hence the subgraph induced by this $G_{i}$ must be transient.

Now, since $G$ is two-ended almost surely, there exist $R$ and $M$ such that, with positive probability, the ball $B_{R}\left(X_{n}\right)$ disconnects $G$ and $\left|B_{R}\left(X_{n}\right)\right| \leq M$. By the Ergodic Theorem this occurs for infinitely many $n$ almost surely. On the event that $X_{n}$ eventually stays in $G_{i}$, since $G_{i}$ is one-ended, this yields an infinite collection of disjoint cutsets of size at most $M$ separating $\rho$ from infinity in $G_{i}$. Thus, $G_{i}$ is recurrent by the Nash-Williams criterion [33], a contradiction.

Theorem 4.2 now follows by combining Theorem 1.1, Corollary 4.5, and Proposition 4.6.

Method 2. This proof reduces Theorem 4.2 to Theorem 1.1 by taking universal covers. Given a (not necessarily planar) map $M$, a cover of $M$ is a map $\tilde{M}$ together with a surjective graph homomorphism $\pi: \tilde{M} \rightarrow M$, such that for each vertex $v$, the homomorphism $\pi$ maps the edges adjacent to $v$ bijectively to the edges adjacent to $\pi(v)$ and preserves their cyclic ordering, and such that for each face $f, \pi$ maps the edges adjacent to $f$ bijectively to the edges adjacent to $\pi(f)$. The universal cover of $M$ is a cover $\pi: \tilde{M} \rightarrow M$ such that $\tilde{M}$ is simply connected. If $M$ is drawn on a surface $S$, the universal cover $\tilde{M}$ of $M$ may be constructed by taking every lift of every edge of $M$ in $S$ to the universal cover $\tilde{S}$ of $S$ (see e.g. [26] for the topological notions of universal cover and path lifting). Alternatively, the universal cover $\tilde{M}$ may be constructed directly as in [41]. The universal cover is unique in the sense that if $\pi^{\prime}: \tilde{M}^{\prime} \rightarrow M$ is also a universal cover of $M$ then there exists an isomorphism of maps $f: \tilde{M}^{\prime} \rightarrow \tilde{M}$ such that $\pi^{\prime}=\pi \circ f$. Note that if a cover $\pi: \tilde{M} \rightarrow M$ is a cover of a map $M$ and $\tilde{M}$ is recurrent, the projection $X_{n}=\pi\left(\tilde{X}_{n}\right)$ of a simple random walk $\tilde{X}_{n}$ on $\tilde{M}$ is a simple random walk on $M$, and it follows that $M$ is also recurrent.

Let $(M, \rho)$ be a unimodular random rooted map with universal cover $\pi: \tilde{M} \rightarrow$ $M$. Let $\tilde{\rho}$ be chosen arbitrarily from the preimage $\pi^{-1}(\rho)$; The isomorphism class of the rooted map $(\tilde{M}, \tilde{\rho})$ does not depend on this choice. We claim that the 
random rooted map $(\tilde{M}, \tilde{\rho})$ is unimodular. To see this, recall that a random rooted graph is unimodular if and only if it is involution invariant [1, Proposition 2.2], meaning that

$$
\mathbb{E} \sum_{v \in V} f(G, \rho, v)=\mathbb{E} \sum_{v \in V} f(G, v, \rho)
$$

whenever $f$ is a mass-transport such that $f(G, u, v)$ is zero unless $u$ and $v$ are adjacent in $G$. The equivalence of unimodularity and involution invariance extends immediately to random rooted maps. Given such an $f: \mathcal{M}_{\bullet \bullet} \rightarrow[0, \infty]$, let $g: \mathcal{M}_{\bullet \bullet} \rightarrow[0, \infty]$ be defined to be

$$
g(M, u, v)=\sum_{e: e^{-}=u, e^{+}=v} f\left(\tilde{M}, \tilde{e}^{-}, \tilde{e}^{+}\right)
$$

where $\pi: \tilde{M} \rightarrow M$ is the universal cover of $M$ and $\tilde{e}$ is an arbitrary element of $\pi^{-1}(e)$ for each oriented edge $e$ of $M$ (by uniqueness of the universal cover, the value of $g$ does not depend on this choice). Then $g$ is a mass transport and, letting $\tilde{V}$ denote the vertex set of $\tilde{M}$, we have

$$
\sum_{v \in V} g(M, \rho, v)=\sum_{\tilde{v} \in \tilde{V}} f(\tilde{M}, \tilde{\rho}, \tilde{v}) \quad \text { and } \quad \sum_{v \in V} g(M, v, \rho)=\sum_{\tilde{v} \in \tilde{V}} f(\tilde{M}, \tilde{v}, \tilde{\rho}),
$$

so that we deduce involution invariance of $(\tilde{M}, \tilde{\rho})$ from involution invariance of $(M, \rho)$. Furthermore, if $(M, \rho)$ is ergodic then $(\tilde{M}, \tilde{\rho})$ is also ergodic. Indeed, for every invariance event $A \subseteq \mathcal{M}_{\bullet}$, the event $\left\{(G, \rho) \in \mathcal{M}_{\bullet}:(\tilde{G}, \tilde{\rho}) \in \mathcal{M}_{\bullet}\right\}$ is also invariant to changing the root, and it follows that if $(M, \rho)$ is ergodic then $(\tilde{M}, \tilde{\rho})$ is also ergodic.

Alternative proof of Theorem 4.2. Let $(G, \rho)$ be a simple, bounded degree, ergodic unimodular random rooted triangulation with $\mathbb{E}[\operatorname{deg}(\rho)]=6$. The universal cover

$(\tilde{G}, \tilde{\rho})$ of $(G, \rho)$ has all these properties and is also one-ended, so that $\tilde{G}$ is $\mathrm{CP}$ parabolic almost surely by Theorem 1.1. By the He-Schramm Theorem [28], $\tilde{G}$ is recurrent almost surely, and so $G$ is also recurrent almost surely, completing the proof of the Benjamini-Schramm Theorem.

\section{Boundary Theory}

Recall that given a $G$ and a vertex $v$ we write $P_{v}^{G}$ and $E_{v}^{G}$ to denote the probability and expectation with respect to random walk $\left(X_{n}\right)_{n \geq 0}$ on $G$ started from $v$. 


\subsection{Convergence to the boundary}

Let $(G, \rho)$ be a one-ended, simple, $\mathrm{CP}$ hyperbolic reversible random triangulation. Recall that for a $\mathrm{CP}$ hyperbolic $G$ with circle packing $\mathcal{C}$ in $\mathbb{D}$, we write $r(v)$ and $z(v)$ for the Euclidean radius and centre of the circle corresponding to the vertex $v$ in $\mathcal{C}$ and $z_{h}(v), r_{h}(v)$ for the hyperbolic centre and radius.

Our first goal is to show that the Euclidean radii $r\left(X_{n}\right)$ decay exponentially along a random walk $\left(X_{n}\right)$. We initially prove only a bound, and will prove the existence of the limit rate of decay stated in Theorem 1.4 only after we have proven the exit measure is non-atomic.

Lemma 5.1. Let $(G, \rho)$ be a $C P$ hyperbolic reversible random rooted triangulation with $\mathbb{E}[\operatorname{deg}(\rho)]<\infty$ and let $\mathcal{C}$ be a circle packing of $G$ in the unit disc. Let $\left(X_{n}\right)_{n \geq 0}$ be a simple random walk on $G$ started from $\rho$. Then almost surely

$$
\limsup _{n \rightarrow \infty} \frac{\log r\left(X_{n}\right)}{n}<0 .
$$

Proof. We may assume that $(G, \rho)$ is ergodic, else we may take an ergodic decomposition. By Theorem $1.1(G, \rho)$ is invariantly non-amenable. By Theorem 3.2, there is an ergodic percolation $\omega$ on $G$ such that $\operatorname{deg}(v)$ is bounded by some $M$ for all $v \in \omega$ and $\mathbf{i}_{E}(\omega)>0$ almost surely.

Recall the notion of an induced random walk on $\omega$ : let $N_{m}$ be the $m$ th time $X$ is in $\omega$ (that is, $N_{0}=\inf \left\{n \geq 0: X_{n} \in \omega\right\}$ and inductively $N_{m+1}=\inf \{n>$ $\left.\left.N_{m}: X_{n} \in \omega\right\}\right)$. The induced network $\bar{\omega}$ is defined to be the weighted graph on the vertices of $\omega$ with edge weights given by

$$
\bar{w}(u, v)=\operatorname{deg}(u) P_{u}^{G}\left(X_{N_{1}}=v\right)
$$

so that $X_{N_{m}}$ is the random walk on the weighted graph $\bar{\omega}$. Note that $\bar{\omega}$ may have non-zero weights between vertices which are not adjacent in $G$, so that $\bar{\omega}$ is no longer a percolation on $G$, and may not even be planar.

We first claim that $\bar{\omega}$ with the weights $\bar{w}$ of the induced random walk also has positive Cheeger constant. Indeed, the weight of a vertex $v \in \bar{\omega}$ is just its degree in $G$ and so is between 1 and $M$ for any vertex. The edge boundary in $\bar{\omega}$ of a set $K$ is at least the number of edges connecting $K$ to $V \backslash K$ in $\omega$. Thus $\mathbf{i}_{E}(\bar{\omega}) \geq \mathbf{i}_{E}(\omega) / M>0$. It follows that the induced random walk on $\omega$ has spectral radius less than one [33].

Now, as in (3.1), Cauchy-Schwarz gives that, for some $c>0$,

$$
P_{\rho}^{G}\left(X_{N_{m}}=v\right) \leq M^{1 / 2} \exp (-c m)
$$

for every vertex $v$ almost surely. Since the total area of all circles in the packing is at most $\pi$, with $c$ as above, there exists at most $e^{c m / 2}$ circles of radius greater 
than $e^{-c m / 4}$ for each $m$. Hence

$$
\begin{aligned}
P_{\rho}^{G}\left(r\left(X_{N_{m}}\right) \geq e^{-c m / 4}\right) & =\sum_{v: r(v) \geq e^{-c m / 4}} P_{\rho}^{G}\left(X_{N_{m}}=v\right) \\
& \leq\left|\left\{v: r(v) \geq e^{-c m / 4}\right\}\right| \cdot M^{1 / 2} e^{-c m} \\
& \leq M^{1 / 2} e^{-c m / 2} .
\end{aligned}
$$

These probabilities are summable, and so Borel-Cantelli implies that almost surely for large enough $m$,

$$
r\left(X_{N_{m}}\right) \leq e^{-c m / 4}
$$

That is, we have exponential decay of the radii for the induced walk:

$$
\limsup _{m \rightarrow \infty} \frac{\log r\left(X_{N_{m}}\right)}{m} \leq-\frac{c}{4}
$$

It remains to prove that the exponential decay is maintained between visits to $\omega$. By stationarity and ergodicity of $(G, \rho, \omega)$, the density of visits to $\omega$ is $\mathbb{P}(\rho \in \omega) \neq 0$. That is,

$$
\lim _{m \rightarrow \infty} \frac{N_{m}}{m}=\mathbb{P}(\rho \in \omega)^{-1}
$$

almost surely. In particular,

$$
\limsup _{m \rightarrow \infty} \frac{\log r\left(X_{N_{m}}\right)}{N_{m}}<0 .
$$

Given $n$, let $m$ be the number of visits to $\omega$ up to time $n$, so that $N_{m} \leq$ $n<N_{m+1}$. Since $m / N_{m}$ converges, $n / N_{m} \rightarrow 1$. By the Sharp Ring Lemma (Theorem 3.7),

$$
\frac{r\left(X_{n}\right)}{r\left(X_{N_{m}}\right)} \leq \exp \left(C \sum_{i=N_{m}}^{n} \operatorname{deg}\left(X_{i}\right)\right)
$$

so that

$$
\frac{\log r\left(X_{n}\right)}{n} \leq \frac{\log r\left(X_{N_{m}}\right)}{n}+\frac{C}{n} \sum_{i=N_{m}}^{n} \operatorname{deg}\left(X_{i}\right) .
$$

Again, the Ergodic Theorem gives us the almost sure limit

$$
\lim \frac{1}{n} \sum_{i=0}^{n} \operatorname{deg}\left(X_{i}\right)=\mathbb{E}[\operatorname{deg}(\rho)]
$$

Thus almost surely

$$
\begin{aligned}
\lim \frac{1}{n} \sum_{i=N_{m}}^{n} \operatorname{deg}\left(X_{i}\right) & =\lim \frac{1}{n} \sum_{i=0}^{n} \operatorname{deg}\left(X_{i}\right)-\lim \frac{N_{m}}{n} \lim \frac{1}{N_{m}} \sum_{i=0}^{N_{m}} \operatorname{deg}\left(X_{i}\right) \\
& =\mathbb{E}[\operatorname{deg}(\rho)]-\mathbb{E}[\operatorname{deg}(\rho)]=0 .
\end{aligned}
$$


Combined with (5.2) and (5.3) we get

$$
\begin{aligned}
\limsup _{n \rightarrow \infty} \frac{\log r\left(X_{n}\right)}{n} & =\limsup _{n \rightarrow \infty} \frac{\log r\left(X_{N_{m}}\right)}{n} \\
& =\lim _{n \rightarrow \infty} \frac{N_{m}}{n} \limsup _{n \rightarrow \infty} \frac{\log r\left(X_{N_{m}}\right)}{N_{m}}<0 .
\end{aligned}
$$

Proof of Theorem 1.3, item 1. We prove the equivalent statement for $(G, \rho)$ reversible with $\mathbb{E}[\operatorname{deg}(\rho)]<\infty$, putting us in the setting of Lemma 5.1. The path formed by drawing straight lines between the Euclidean centres of the circles along the random walk path has length $r(\rho)+2 \sum_{i \geq 1} r\left(X_{i}\right)$, which is almost surely finite by Lemma 5.1. It follows that the sequence of Euclidean centres is Cauchy almost surely and hence converges to some point, necessarily in the boundary. Because the radii of the circles $r\left(X_{n}\right)$ converge to zero almost surely, the hyperbolic centres must also converge to the same point.

\subsection{Full support and non-atomicity of the exit measure}

We now prove item 2 of Theorem 1.3, which states that the exit measure on the unit circle has full support and no atoms almost surely. We start with a general observation regarding atoms in boundaries of stationary graphs.

We say that two metrics $d_{1}$ and $d_{2}$ on the vertex set $V$ of a graph $G$ are compatible if the identity map from $V$ to itself extends to an isomorphism between the completions of the metric spaces $\left(V, d_{1}\right)$ and $\left(V, d_{2}\right)$ (or, equivalently, if the same sequences are Cauchy for $d_{1}$ and $\left.d_{2}\right)$. For example, the Euclidean distances between centres of circles corresponding to vertices in different circle packings of a one-ended planar triangulation in either the full plane or the unit disc are compatible by Theorem 3.5. We define a compatible family of metrics to be a Borel function $d=d_{u}^{G}(v, w)$ from the space of triply rooted graphs (i.e., the set of isomoprhism classes of graphs with an ordered triple of distinguished vertices, equipped with an appropriate variant of the local topology) to the positive reals such that for every locally finite, connected graph $G=(V, E)$,

1. $d_{u}^{G}(\cdot, \cdot)$ is a metric on $V$ for every vertex $u$ of $G$, and

2. the metrics $d_{u}^{G}$ and $d_{v}^{G}$ are compatible for each two vertices $u$ and $v$ of $G$.

Given a compatible family of metrics $d$ and a rooted graph $(G, \rho)$, the completion $\bar{V}$ of $V$ with respect to $d_{\rho}^{G}$ has a topology that does not depend on the choice of root vertex $\rho$. Such a completion is called an invariant completion. Compatible families of metrics for maps are defined similarly. In some cases of interest, a compatible family of metrics $d$ might only be defined for graphs or maps in some rerooting-invariant class (e.g. circle packings are defined for the class of one-ended 
simple planar triangulations). In this case, we may extend $d$ arbitrarily to all graphs or maps by setting it to be the discrete metric where it is not defined.

Lemma 5.2. Let d be a compatible family of metrics, let $(G, \rho)$ be a stationary random rooted graph or map, and let $\bar{V}$ be the completion of $V$ with respect to $d_{\rho}^{G}$. Suppose that the random walk on $G$ converges almost surely to a point in the boundary $\partial V=\bar{V} \backslash V$. Then the exit measure on $\partial V$ is either trivial (concentrated on a single point) or non-atomic almost surely.

For each CP hyperbolic one-ended simple planar triangulation $G$, take a circle packing of $G$ in $\mathbb{D}$, normalized so that the circle corresponding to $u$ is centred at the origin, and let $d=d_{u}^{G}(v, w)$ be the Euclidean distance between the Euclidean centres of the circles corresponding to $v$ and $w$. By circle packing rigidity (Theorem 3.5), this circle packing is unique up to rotations, so that the metric $d_{u}^{G}$ is well defined. The metrics $d_{u}^{G}$ and $d_{v}^{G}$ are also compatible for every pair of vertices $u$ and $v$ in $G$. Thus, after an arbitrary extension to other maps, $d$ is an compatible family of metrics.

Another natural example, defined for all graphs, is the Martin compactification. As a consequence of this lemma, for any stationary random graph, the exit measure on the Martin boundary (which can be defined as the completion of $V$ with respect to a compatible family of metrics, see e.g. [44]) is almost surely either non-atomic or trivial. In particular, this gives an alternative proof of a recent result of Benjamini, Paquette and Pfeffer [15], which states that for every stationary random graph, the space of bounded harmonic functions on the graph is either one dimensional or infinite dimensional almost surely. A straightforward extension of this lemma applies to random families of metrics.

Proof. Condition on $(G, \rho)$. For each atom $\xi$ of the exit measure, define the harmonic function $h_{\xi}(v)=P_{v}^{G}\left(\lim X_{n}=\xi\right)$. By Lévy's 0-1 law,

$$
h_{\xi}\left(X_{n}\right) \stackrel{\text { a.s. }}{\longrightarrow} \mathbb{1}\left(\lim X_{n}=\xi\right)
$$

for each atom $\xi$ and

$$
P_{X_{n}}^{G}\left(\lim X_{n} \text { is an atom }\right)=\sum_{\xi} h_{\xi}\left(X_{n}\right) \stackrel{\text { a.s. }}{\longrightarrow} \mathbb{1}\left(\lim X_{n} \text { is an atom }\right) .
$$

Define $M(G, v)=\max _{\xi} h_{\xi}(v)$ to be the maximal atom size. Since the topology of $\bar{V}$ does not depend on the choice of root, the sequence $M\left(G, X_{n}\right)$ is stationary. Combining the two above limits, we find the almost sure limit

$$
M\left(G, X_{n}\right) \stackrel{\text { a.s. }}{\longrightarrow} \mathbb{1}\left(\lim X_{n} \text { is an atom }\right) .
$$

Since $M\left(G, X_{n}\right)$ is a stationary sequence with limit in $\{0,1\}$, it follows that $M(G, \rho) \in\{0,1\}$ almost surely. That is, either there are no atoms in the exit measure or there is a single atom with weight 1 almost surely. 
Proof of Theorem 1.3, item 2. We may assume that $(G, \rho)$ is ergodic. Applying Lemma 5.2 to the $\operatorname{deg}(\rho)$-biasing of $(G, \rho)$, we deduce that the exit measure has at most a single atom. Next, we rule out having a single atom. Suppose for contradiction that there is a single atom $\xi=\xi(\mathcal{C})$ almost surely for some (and hence every) circle packing $\mathcal{C}$ of $G$ in $\mathbb{D}$. Applying the Möbius transformation

$$
\Phi(z)=-i \frac{z+\xi}{z-\xi}
$$

which maps $\mathbb{D}$ to the upper half-plane $\mathbb{H}=\{\Im(z)>0\}$ and $\xi$ to $\infty$, gives a circle packing of $G$ in $\mathbb{H}$ such that the random walk tends to $\infty$ almost surely. Since circle packings in $\mathbb{H}$ are unique up to Möbius transformations and the boundary point $\infty$ is determined by the graph $G$, such a circle packing in $\mathbb{H}$ is unique up to Möbius transformations of the upper half-plane that fix $\infty$, namely $a z+b$ with real $a \geq 0$ and $b$ (translations and dilations).

Inverting around the atom has therefore given us a way of canonically endowing $G$ with Euclidean geometry: if we draw $G$ in $\mathbb{H}$ using straight lines between the Euclidean centres of the circles in the half-plane packing, the angles at the corners around each vertex $u$ are independent of the original choice of packing $\mathcal{C}$. Transporting each angle from $u$ to each of the three vertices forming the corresponding face $f$ as in the proof of Theorem 1.1 implies that $\mathbb{E}[\operatorname{deg}(\rho)]=6$. This contradicts Theorem 1.1 and the assumption that $(G, \rho)$ is CP hyperbolic almost surely. This completes the proof that the exit measure is non-atomic.

To finish, we show that the exit measure has support $\partial \mathbb{D}$. Suppose not. We will define a mass transport on $G$ in which each vertex sends a mass of at most one but some vertices receive infinite mass, contradicting the mass transport principle.

Consider the complement of the support of the exit measure, which is a union of disjoint open intervals $\bigcup_{i \in I}\left(\theta_{i}, \psi_{i}\right)$ in $\partial \mathbb{D}$. Since the exit measure is non-atomic, $\theta_{i} \neq \psi_{i} \bmod 2 \pi$ for all $i$.

For each such interval $\left(\theta_{i}, \psi_{i}\right)$, let $\gamma_{i}$ be the hyperbolic geodesic from $e^{i \theta_{i}}$ to $e^{i \psi_{i}}$. That is, $\gamma_{i}$ is the intersection with $\mathbb{D}$ of the circle passing through both $e^{i \theta_{i}}$ and $e^{i \psi_{i}}$ that intersects $\partial \mathbb{D}$ at right angles. Let $A_{i}$ be the set of vertices such that the circle corresponding to $v$ is contained in the region to the right of $\gamma_{i}$, i.e. bounded between $\gamma_{i}$ and the boundary interval $\left(\theta_{i}, \psi_{i}\right)$ (see Figure $3(\mathrm{a})$ ).

Each vertex is contained in at most one such $A_{i}$. For each vertex $u$ in $A_{i}$, consider the hyperbolic geodesic ray $\gamma_{u}$ from the hyperbolic centre $z_{h}(u)$ to $e^{i \theta_{i}}$. Define a mass transport by sending mass one from $u \in A_{i}$ to the vertex $v$ corresponding to the first circle intersected by both $\gamma_{u}$ and $\gamma_{i}$. There may be no such circle, in which case no mass is sent from $u$. Since the transport is defined in terms of the hyperbolic geometry and the support of the exit measure, it is a function of the isomorphism class of $(G, u, v)$ by Theorem 3.5. 


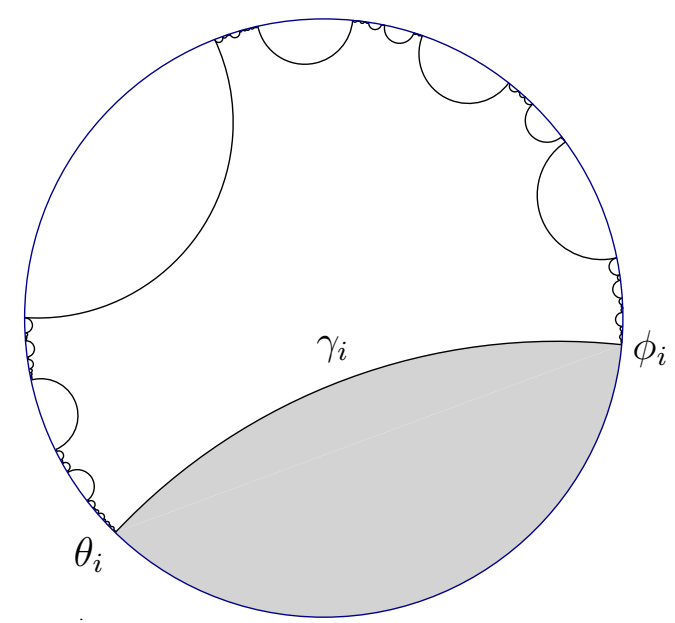

(a) A geodesic $\gamma_{i}$ is drawn over each component of the complement of the support. Circles contained in the shaded area are in $A_{i}$.

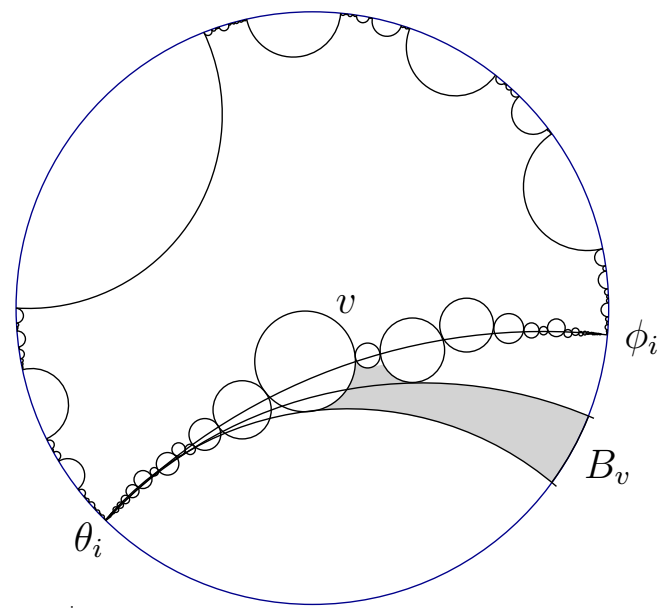

(b) The vertex $v$ receives mass from circles with hyperbolic centres in the shaded area.

Figure 3: An illustration of the mass transport used to show the exit measure has full support.

Let $\phi \in\left(\theta_{i}, \psi_{i}\right)$ and consider the set of vertices whose corresponding circles intersect both $\gamma_{i}$ and the geodesic $\gamma_{\phi}$ from $e^{i \phi}$ to $e^{i \theta_{i}}$. As $\phi$ increases from $\theta_{i}$ to $\psi_{i}$, this set is increasing. It follows that for each fixed $v$ for which the circle corresponding to $v$ intersects $\gamma_{i}$, the set $B_{v}$ of $\phi \in\left(\theta_{i}, \psi_{i}\right)$ for which the circle corresponding to $v$ is the first circle intersected by $\gamma_{\phi}$ that also intersects $\gamma_{i}$ is an interval (see Figure 3(b)).

Since there are only countably many vertices, $B_{v}$ must have positive length for some $v$. Thus there is an open neighbourhood of the boundary in which all the circles send mass to this vertex. This vertex therefore receives infinite mass, contradicting the mass transport principle.

\subsection{The unit circle is the Poisson boundary}

The Poisson-Furstenberg boundary [22] (or simply the Poisson boundary) of a graph (or more generally, of a Markov chain) is a formal way to encode the asymptotic behaviour of random walks on $G$. We refer the reader to $[29,35,33]$ for more detailed introductions. 
Recall that a function $h: V(G) \rightarrow \mathbb{R}$ is said to be harmonic if

$$
h(v)=\frac{1}{\operatorname{deg}(v)} \sum_{u \sim v} h(u)
$$

for all $v \in V(G)$ - or, equivalently, if $h\left(X_{n}\right)$ is a martingale. Let $\bar{G}=G \cup \partial G$ be a compactification of $G$ so that the random walk $X_{n}$ converges almost surely. For each $v \in V(G)$ we let $P_{v}^{G}$ denote the law of the limit of the random walk started at $v$. Every bounded Borel function $g$ on $\partial G$ extends to a harmonic function

$$
h(v):=E_{v}^{G}\left[g\left(\lim X_{n}\right)\right]
$$

on $G$. Such a compactification is called a realisation of the Poisson boundary of $G$ if every bounded harmonic funtion $h$ on $G$ may be represented as an extension of a boundary function in this way.

Harmonic functions can be used to encode asymptotic behaviour of the random walk as follows. Let $G^{\mathbb{N}}$ be the space of sequences in $G$. The shift operator on $G^{\mathbb{N}}$ is defined by $\theta\left(x_{0}, x_{1}, \ldots\right)=\left(x_{1}, x_{2}, \ldots\right)$, and we write $\mathcal{I}$ for the $\sigma$-algebra of shift-invariant events $A=\theta A$. Be careful to note the distinction between invariant events for the random walk on $G$, just defined, and invariant events for the sequence $\left(G,\left(X_{n+k}\right)_{n \in \mathbb{Z}}\right)_{k \in \mathbb{Z}}$ as defined in Section 3.2.

There is an isomorphism between the space of bounded harmonic functions on $G$ and $L^{\infty}\left(G^{\mathbb{N}}, \mathcal{I}\right)$ given by

$$
h \mapsto g\left(x_{1}, x_{2}, \ldots\right)=\lim _{n \rightarrow \infty} h\left(x_{n}\right), \quad g \mapsto h(v)=E_{v}^{G}\left[g\left(v, X_{1}, X_{2}, \ldots\right)\right] .
$$

The limit here exists $P_{v}^{G}$-almost surely by the bounded Martingale Convergence Theorem, while the fact that these two mappings are inverses of one another is a consequence of Lévy's 0-1 Law: If $h(v)=E_{v}^{G}[g(X)]$ is the harmonic extension of some invariant function $g$, then

$$
h\left(X_{n}\right) \stackrel{\text { a.s. }}{\longrightarrow} g\left(\rho, X_{1}, X_{2}, \ldots\right) .
$$

As a consequence of this isomorphism, and since the span of simple functions is dense in $L^{\infty}$, the topological boundary $\partial G$ is a realisation of the Poisson boundary of $G$ if and only if for every invariant event $A$ there exists a Borel set $B \subset \partial G$ such that the symmetric difference $A \Delta\left\{\lim X_{n} \in B\right\}$ is $P_{v}^{G}$-null.

For example, the Poisson boundary of a tree may be realised as its space of ends, and the one-point compactification of a transient graph $\mathrm{G}$ gives rise to a realisation of the Poisson boundary if and only if $G$ is Liouville (i.e. the only bounded harmonic functions on $G$ are constant). The Poisson boundary of any graph may be realised as the graph's Martin boundary [44], but this is not always the most natural construction. 
Our main tools for controlling harmonic functions will be Lévy's 0-1 Law and the following consequence of the Optional Stopping Theorem. For a set $W \subset V$ of vertices, let $T_{W}$ be the first time the random walk visits $W$. If $h$ is a positive, bounded harmonic function, the Optional Stopping Theorem implies

$$
h(v) \geq E_{v}^{G}\left[h\left(X_{T_{W}}\right) \mathbb{1}_{T_{W}<\infty}\right] \geq P_{v}^{G}(\text { Hit } W) \inf \{h(u): u \in W\} .
$$

Lemma 5.3. Let $(G, \rho)$ be a $C P$ hyperbolic reversible random rooted triangulation with $\mathbb{E}[\operatorname{deg}(\rho)]<\infty$, and let $\left(X_{n}\right)_{n \in \mathbb{Z}}$ be the reversible bi-infinite random walk. Then almost surely

$$
P_{X_{n}}^{G}\left(\text { hit }\left\{X_{-1}, X_{-2}, \ldots\right\}\right) \rightarrow 0 .
$$

Proof. Let $\mathcal{C}$ be a circle packing of $G$ in $\mathbb{D}$. Recall from Theorem 1.3, item 1 that for a random walk $X_{n}$, almost surely $\Xi:=\lim z\left(X_{n}\right)$ exists, and its law is non-atomic and of full support on $\partial \mathbb{D}$. Since the exit measure is non-atomic, the limit points $\Xi_{+}:=\lim z\left(X_{n}\right)$ and $\Xi_{-}:=\lim z\left(X_{-n}\right)$ are almost surely distinct.

Let $\left\{U_{i}\right\}_{i \in I}$ be a countable basis for the topology of $\partial \mathbb{D}$ (say, intervals with rational endpoints) and for each $i$ let $h_{i}$ be the harmonic function

$$
h_{i}(v)=P_{v}^{G}\left(\Xi \in U_{i}\right) .
$$

By Lévy's 0-1 law, $h_{i}\left(X_{n}\right) \rightarrow \mathbb{1}\left(\Xi_{+} \in U_{i}\right)$ for every $i$ almost surely. Thus there exists some $i_{0}$ with $\Xi_{-} \in U_{i_{0}}$ and $\Xi_{+} \notin U_{i_{0}}$. In particular there is almost surely some bounded harmonic function $h=h_{i_{0}} \geq 0$ with $h\left(X_{n}\right) \underset{n \rightarrow \infty}{\longrightarrow} 0$ and

$$
a:=\inf \left\{h\left(X_{-m}\right): m>0\right\}>0 .
$$

By (5.5)

$$
h\left(X_{n}\right) \geq a \cdot P_{X_{n}}^{G}\left(\text { hit }\left\{X_{-1}, X_{-2}, \ldots\right\}\right) .
$$

Since $h\left(X_{n}\right) \rightarrow 0$, we almost surely have

$$
P_{X_{n}}^{G}\left(\text { Hit }\left\{X_{-1}, X_{-2}, \ldots\right\}\right) \rightarrow 0 .
$$

Proof of Theorem 1.3, item 3. We prove the equivalent statement for $(G, \rho)$ reversible with $\mathbb{E}[\operatorname{deg}(\rho)]<\infty$, and may assume that $(G, \rho)$ is ergodic.

We need to prove that for every invariant event $A$ for the simple random walk on $G$ with $P_{\rho}^{G}(A)>0$, there is a Borel set $B \subset \partial \mathbb{D}$ such that

$$
P_{\rho}^{G}\left(A \Delta\left\{\Xi_{+} \in B\right\}\right)=0,
$$

where $\Xi_{+}=\lim z\left(X_{n}\right)$. Let $h$ be the harmonic function $h(v)=P_{v}^{G}(A)$, and let $B$ be the set of $\xi \in \partial \mathbb{D}$ such that there exists a path $\left(\rho, v_{1}, v_{2}, \ldots\right)$ in $G$ such that for some $c>0$,

$$
h\left(v_{i}\right) \rightarrow 1, \quad z\left(v_{i}\right) \rightarrow \xi, \quad \text { and } \quad\left|\xi-z\left(v_{i}\right)\right|<2 e^{-c i},
$$




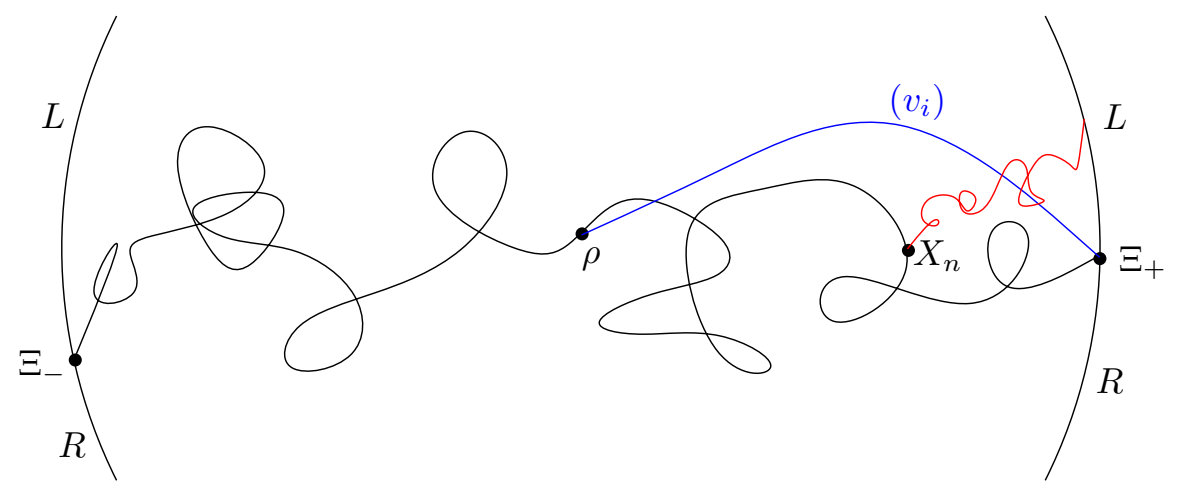

Figure 4: For infinitely many $n$, a new random walk (red) started from $X_{n}$ has probability at least $1 / 3$ of hitting each of $L$ and $R$, and probability at least $1 / 4$ of hitting the path $\left(v_{i}\right)$ (blue).

where $|\cdot|$ denotes Euclidean length. The condition on exponential decay of $\left|\xi-z\left(v_{i}\right)\right|$ can be omitted by invoking the theory of universally measurable sets. We are spared from this by Lemma 5.1. With an explicit rate of convergence, it is straightforward to see that $B$ is Borel: Let $B_{c, m, \varepsilon, n}$ be the open set of $\xi \in \partial \mathbb{D}$ such that there exists a path $\rho, v_{1}, \ldots, v_{n}$ in $G$ such that $h\left(v_{i}\right)>1-\varepsilon$ for every $i \geq m$, and with $\left|\xi-z\left(v_{i}\right)\right|<2 e^{-c i}$. Then $B=\bigcup_{c} \bigcap_{\varepsilon} \bigcup_{m} \bigcap_{n} B_{c, m, \varepsilon, n}$, where $m, n$ are integers and $c, \varepsilon$ are positive rationals, and it follows that $B$ is Borel.

If the random walk has $\left(\rho, X_{1}, \ldots\right) \in A$ then, by Lévy's 0-1 law and Lemma 5.1, the limit point $\Xi_{+}$is in $B$ almost surely. In particular, if $P_{\rho}^{G}(A)>0$, then the exit measure of $B$ is positive. It remains to show that $\left(\rho, X_{1}, \ldots\right) \in A$ almost surely on the event that $\Xi_{+} \in B$.

Consider the two intervals $L$ and $R$ separating the almost surely distinct limit points $\Xi_{+}$and $\Xi_{-}$. Let $p_{L}^{n}$ and $p_{R}^{n}$ be the probabilities that a new, independent random walk started from $X_{n}$ hits the boundary in the interval $L$ or $R$ respectively. Since the exit measure is non-atomic almost surely, the event

$$
E_{n}=\left\{\min \left(p_{L}^{n}, p_{R}^{n}\right)>1 / 3\right\}
$$

has positive probability (in fact, it is not hard to see that each of the random variables $p_{L}^{n}$ is uniformly distributed on $[0,1]$ so that $E_{n}$ has probability $1 / 3$ ). Moreover, the value of $p_{L}^{n}$ does not depend on the choice of circle packing and is therefore a function of $\left(G,\left(X_{n+k}\right)_{k \in \mathbb{Z}}\right)$. By the stationarity and ergodicity of $\left(G,\left(X_{n}\right)_{n \in \mathbb{Z}}\right)$, the events $E_{n}$ happen infinitely often almost surely (see Figure 4$)$.

Now condition on $\Xi_{+} \in B$. Since the exit measure of $B$ is positive, the events $E_{n}$ still happen infinitely often almost surely after conditioning. Let $\left(v_{i}\right)_{i \geq 0}$ be a path from $\rho$ in $G$ such that $z\left(v_{i}\right) \rightarrow \Xi_{+} \in B$ and $h\left(v_{i}\right) \rightarrow 1$. In particular,

$$
\inf \left\{h\left(v_{i}\right): i \geq 1\right\}>0 \text {. }
$$


The path $\left(\ldots, X_{-2}, X_{-1}, \rho, v_{1}, v_{2}, \ldots\right)$ disconnects $X_{n}$ from at least one of the intervals $L$ or $R$ and so

$$
P_{X_{n}}^{G}\left(\text { hit }\left\{\ldots, X_{-1}, \rho, v_{1}, \ldots\right\}\right) \geq \min \left(p_{L}^{n}, p_{R}^{n}\right)
$$

which is greater than $1 / 3$ infinitely often almost surely. We stress that the expression refers to the probability that an independent random walk started from $X_{n}$ hits the path $\left(\ldots, X_{-1}, \rho, v_{1}, \ldots\right)$, and that this bound holds trivially if $X_{n}$ is on the path $\left(v_{m}\right)$. By Lemma 5.3,

$$
P_{X_{n}}^{G}\left(\text { hit }\left\{\ldots, X_{-1}, \rho\right\}\right) \stackrel{\text { a.s. }}{\longrightarrow} 0 \text {, }
$$

and hence $P_{X_{n}}^{G}$ (hit $\left.\left\{v_{1}, v_{2}, \ldots\right\}\right)>1 / 4$ infinitely often almost surely (see Figure 4 ). Note that, since the choice of $v_{i}$ could depend on the whole trajectory of $X$, we have not shown that $X$ hits the path $\left(v_{i}\right)$ infinitely often. Nevertheless, by (5.5), almost surely infinitely often

$$
h\left(X_{n}\right)>\frac{1}{4} \inf \left\{h\left(v_{i}\right): i \geq 1\right\}>0 .
$$

By Lévy's 0-1 law, $\lim _{n \rightarrow \infty} h\left(X_{n}\right)=1$ almost surely as desired.

\section{Hyperbolic speed and decay of radii}

We now use the fact that the exit measure is almost surely non-atomic to strengthen Lemma 5.1 and deduce that the limit rate of decay of the Euclidean radii along the random walk exists. The key idea is to use a circle packing in the upper half-plane normalised by the limits of two independent random walks.

Fix some circle packing $\mathcal{C}$ in $\mathbb{D}$, so that, by Theorem 1.3, the limit points $\Xi_{ \pm}=\lim _{n \rightarrow \pm \infty} z\left(X_{n}\right)$ exist and are distinct almost surely. Let $\Phi_{X}$ be a Möbius transformation that maps $\mathbb{D}$ to the upper half-plane $\mathbb{H}$ and sends $\Xi_{+}$to 0 and $\Xi_{-}$ to $\infty$. We consider the upper half-plane packing $\widehat{\mathcal{C}}=\Phi_{X}(\mathcal{C})$.

Similarly to the proof of non-atomicity in Section 5.2, we now have two boundary points 0 and $\infty$ fixed by the graph $G$ and the path $\left(X_{n}\right)$, so that the resulting circle packing is unique up to scaling. Now, however, the packing depends on both $G$ and the random walk, so that this new situation is not paradoxical (as it was in Section 5.2 where we ruled out the possibility that the exit measure has a single atom).

Proof of Theorem 1.4. We prove the equivalent statement for $(G, \rho)$ reversible with $\mathbb{E}[\operatorname{deg}(\rho)]<\infty$, and may assume that $(G, \rho)$ is ergodic. We fix a circle packing $\widehat{\mathcal{C}}=\Phi_{X}(\mathcal{C})$ in $\mathbb{H}$ as above, with the doubly infinite random walk from 
$\infty$ to 0 . Let $\hat{r}(v)$ be the Euclidean radius of the circle corresponding to $v$ in $\widehat{\mathcal{C}}$. The ratio of radii $\hat{r}\left(X_{n}\right) / \hat{r}\left(X_{n-1}\right)$ does not depend on the choice of $\widehat{\mathcal{C}}$, so these ratios form a stationary ergodic sequence. By the Sharp Ring Lemma, $\mathbb{E}\left[\left|\log \left(\hat{r}\left(X_{1}\right) / \hat{r}(\rho)\right)\right|\right] \leq C \mathbb{E}[\operatorname{deg}(\rho)]<\infty$, so that the Ergodic Theorem implies that

$$
-\frac{1}{n} \log \frac{\hat{r}\left(X_{n}\right)}{\hat{r}(\rho)}=-\frac{1}{n} \sum_{1}^{n} \log \frac{\hat{r}\left(X_{i}\right)}{\hat{r}\left(X_{i-1}\right)} \underset{n \rightarrow \infty}{\stackrel{a . s .}{\longrightarrow}}-\mathbb{E}\left[\log \frac{\hat{r}\left(X_{1}\right)}{\hat{r}(\rho)}\right] .
$$

Now, since $\widehat{\mathcal{C}}$ is the image of $\mathcal{C}$ through the Möbius map $\Phi_{X}$, and since $\Phi_{X}$ is conformal at $\Xi_{+}$,

$$
\frac{\hat{r}\left(X_{n}\right)}{r\left(X_{n}\right)} \rightarrow\left|\Phi_{X}^{\prime}\left(\Xi_{+}\right)\right|>0
$$

Therefore

$$
\lim \frac{-\log r\left(X_{n}\right)}{n}=\mathbb{E}\left[-\log \frac{\hat{r}\left(X_{1}\right)}{\hat{r}(\rho)}\right]
$$

and by Lemma 5.1 this limit must be positive. This establishes the rate of decay of the radii.

Next, we relate this to the distance of $z\left(X_{n}\right)$ from $\partial \mathbb{D}$. By the triangle inequality, $1-\left|z\left(X_{n}\right)\right|$ is at most the length of the path formed by drawing straight lines between the Euclidean centres of the circles along the random walk path starting at $X_{n}$ :

$$
1-\left|z_{h}\left(X_{n}\right)\right| \leq 1-\left|z\left(X_{n}\right)\right| \leq \sum_{i \geq n} 2 r\left(X_{i}\right)
$$

Since the radii decay exponentially, taking the limits of the logarithms,

$$
\liminf \frac{-\log \left(1-\left|z_{h}\left(X_{n}\right)\right|\right)}{n} \geq \lim \frac{-\log r\left(X_{n}\right)}{n} .
$$

To get a corresponding upper bound, note that, since every circle neighbouring $X_{n}$ is contained in the open unit disc, $1-\left|z_{h}\left(X_{n}\right)\right|$ is at least the radius of the smallest neighbour of $X_{n}$. Applying the Sharp Ring Lemma, we have

$$
1-\left|z_{h}\left(X_{n}\right)\right| \geq r\left(X_{n}\right) \exp \left(-C \operatorname{deg}\left(X_{n}\right)\right) \text {. }
$$

Taking logarithms and passing to the limit,

$$
\begin{aligned}
\limsup \frac{-\log \left(1-\left|z_{h}\left(X_{n}\right)\right|\right)}{n} & \leq \lim \frac{-\log r\left(X_{n}\right)}{n}+\lim \frac{C \operatorname{deg}\left(X_{n}\right)}{n} \\
& =\lim \frac{-\log r\left(X_{n}\right)}{n},
\end{aligned}
$$


where the almost sure limit $\operatorname{deg}\left(X_{n}\right) / n \rightarrow 0$ follows from $\mathbb{E}[\operatorname{deg}(\rho)]<\infty$ and Borel-Cantelli. Combining (6.4) and (6.5) gives the almost sure limit

$$
\lim \frac{-\log \left(1-\left|z_{h}\left(X_{n}\right)\right|\right)}{n}=\lim \frac{-\log r\left(X_{n}\right)}{n} .
$$

Finally, to relate this to the speed in the hyperbolic metric, recall that distances from the origin in the hyperbolic metric are given by

$$
d_{\text {hyp }}(0, z)=2 \tanh ^{-1}|z|
$$

and hence

$$
\begin{aligned}
\lim \frac{1}{n} d_{\text {hyp }}\left(z_{h}(\rho), z_{h}\left(X_{n}\right)\right) & =\lim \frac{1}{n} d_{\text {hyp }}\left(0, z_{h}\left(X_{n}\right)\right) \\
& =\lim \frac{2}{n} \tanh ^{-1}\left|z_{h}\left(X_{n}\right)\right| \\
& =\lim -\frac{1}{n} \log \left(1-\left|z_{h}\left(X_{n}\right)\right|\right) .
\end{aligned}
$$

\section{Extensions}

We now discuss two basic extensions of our main results beyond simple triangulations. These are to weighted and to non-simple triangulations. The latter are of particular interest since the PSHT is not simple. Some of our results hold for much more general planar maps, which are treated in [7].

Weighted networks. Suppose $(G, \rho, w)$ is a unimodular random rooted weighted triangulation. As in the unweighted case, if $\mathbb{E}[w(\rho)]$ is finite then biasing by $w(\rho)$ gives an equivalent random rooted weighted triangulation which is reversible for the weighted simple random walk [1, Theorem 4.1]. Our arguments generalise with no change to recover all our main results in the weighted setting provided the following conditions are satisfied.

1. $\mathbb{E}[w(\rho)]<\infty$. This allows us to bias to get a reversible random rooted weighted triangulation.

2. $\mathbb{E}[w(\rho) \operatorname{deg}(\rho)]<\infty$. After biasing by $w(\rho)$, the expected degree is finite, allowing us to apply the Ring Lemma together with the Ergodic Theorem as in the proofs of Lemma 5.1 and Theorem 1.4.

3. A version of Theorem 3.2 holds. That is, there exists a percolation $\omega$ such that the induced network $\bar{\omega}$ has positive Cheeger constant almost surely. Two natural situations in which this occurs are 

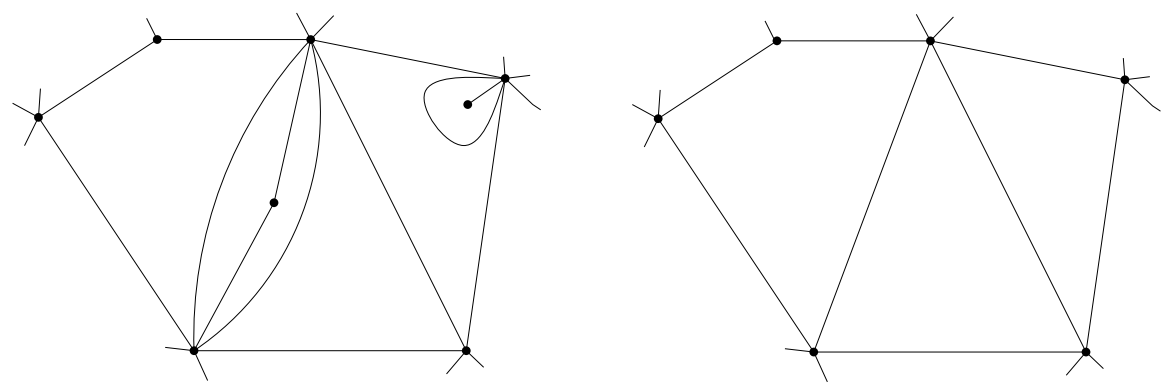

Figure 5: Extracting the core of a non-simple map. Left: part of a map. Right: corresponding part of its core.

(a) when all the weights are non-zero almost surely. In this situation, we may adapt the proof of Theorem 3.2 by first deleting all edges of weight less than $1 / M$ and all vertices of total weight greater than $M$ before continuing the construction as before.

(b) when the subgraph formed by the edges of non-zero weight is connected and is itself invariantly non-amenable. This occurs when we circle pack planar maps that are not triangulations by adding edges of weight 0 in non-triangular faces to triangulate them.

Non-simple triangulations. Suppose $G$ is a one-ended planar map. The endpoints of any double edge or loop in $G$ disconnect $G$ into connected components exactly one of which is infinite. The simple core of $G$, denoted core $(G)$, is defined by deleting the finite component contained within each double edge or loop of $G$ before gluing the double edges together or deleting the loop as appropriate. See Figure 5 for an example, and [10] for a more detailed description. When $G$ is a triangulation, so is its core. The core can be seen as a subgraph of $G$, with some vertices removed, and multiple edges replaced by a single edge. The induced random walk on the core, is therefore a random walk on a weighted simple triangulation.

In general, it is possible that all of $G$ is deleted by this procedure, but in this case there are infinitely many disjoint vertex cut-sets of size 2 separating each vertex from infinity, implying that $G$ is VEL parabolic and hence invariantly amenable. When $G$ is invariantly non-amenable, the conclusions of Theorem 1.3 hold with the necessary modifications.

Theorem 7.1. Let $(G, \rho)$ be an invariantly non-amenable, one-ended, unimodular random rooted planar triangulation with $\mathbb{E}\left[\operatorname{deg}^{2}(\rho)\right]<\infty$. Then $\operatorname{core}(G)$ is $C P$ hyperbolic. Let $\mathcal{C}$ be a circle packing of $\operatorname{core}(G)$ in $\mathbb{D}$, and let $\left(Y_{n}\right)_{n \in \mathbb{N}}$ be the induced random walk on $\operatorname{core}(G)$. The following hold conditional on $(G, \rho)$ almost surely: 
1. $z\left(Y_{n}\right)$ and $z_{h}\left(Y_{n}\right)$ both converge to a (random) point denoted $\Xi \in \partial \mathbb{D}$,

2. The law of $\Xi$ has full support and no atoms.

3. $\partial \mathbb{D}$ is a realisation of the Poisson boundary of $G$. That is, for every bounded harmonic function $h$ on $G$ there exists a bounded measurable function $g$ : $\partial \mathbb{D} \rightarrow \mathbb{R}$ such that

$$
h(v)=E_{v}^{G}[g(\Xi)] .
$$

Since the additional components needed to prove this are straightforward, we omit some of the details.

Sketch of proof. First, $(\operatorname{core}(G), \rho)$ is unimodular when sampled conditional on $\rho \in \operatorname{core}(G)$ : essentially, a mass transport on core $(G)$ gives a mass transport on $G$ which is 0 for all deleted vertices. The mass transport principle for $G$ implies the principle for $\operatorname{core}(G)$.

Second, core $(G)$ is CP hyperbolic. Since $(G, \rho)$ is invariantly non-amenable, it is VEL hyperbolic (see the proof of Theorem 1.1). Because the infimum over paths in the definition of the vertex extremal length is the same as the infimum over paths in the core, the vertex extremal length from $v \in \operatorname{core}(G)$ to $\infty$ is the same in $G$ and $\operatorname{core}(G)$. (Alternatively, one could deduce non-amenability of $\operatorname{core}(G)$ from non-amenability of $G$, and apply Theorem 1.1.)

Now, since $\operatorname{core}(G)$ is a weighted CP hyperbolic unimodular simple triangulation (and the second moment of the degree of the root is finite), by Theorem 1.3 the random walk on $\operatorname{core}(G)$ converges to a point in the boundary, the exit measure has full support and no atoms, and $\partial \mathbb{D}$ is a realisation of the Poisson boundary of $\operatorname{core}(G)$.

Finally, by the Optional Stopping Theorem, the bounded harmonic functions on core $(G)$ are in one-to-one correspondence with the bounded harmonic functions on $G$ by restriction and extension:

$$
h_{G} \mapsto h_{\operatorname{core}(G)}=\left.h_{G}\right|_{\operatorname{core}(G)}, \quad h_{\operatorname{core}(G)} \mapsto h_{G}(v)=E_{v}^{G}\left[h_{\operatorname{core}(G)}\left(X_{N_{0}}\right)\right] .
$$

Thus, the realisation of $\partial \mathbb{D}$ as the Poisson boundary of core $(G)$ extends to $G$.

\section{Open Problems}

Problem 8.1. Can the identification of the Poisson and geometric boundaries be strengthened to an identification of the Martin boundary? This was done in [4] for CP hyperbolic triangulations with bounded degrees. Specifically, we believe the following. 
Conjecture 8.2. Let $(G, \rho)$ be an infinite simple, one-ended, CP hyperbolic unimodular random rooted planar triangulation with $\mathbb{E}[\operatorname{deg}(\rho)]<\infty$, and let $\mathcal{C}$ be a circle packing of $G$ in the unit disc. Then almost surely for every point $\xi \in \partial \mathbb{D}$ there exists a unique positive harmonic function $h_{\xi}$ on $G$ such that $h_{\xi}(\rho)=1$ and $h_{\xi}$ is bounded on $\{v:|z(v)-\xi| \geq \varepsilon\}$ for every $\varepsilon>0$. Moreover, the function $\xi \mapsto h_{\xi}$ almost surely extends to a homeomorphism from $z(V) \cup \partial \mathbb{D}$ to the Martin compactification of $G$.

Problem 8.3 (Hölder continuity of the exit measures). In the setting of Theorem 1.3, do there exist positive constants $c$ and $C$ such that

$$
P_{\rho}^{G}(\Xi \in I) \leq C|I|^{c}
$$

for every interval $I \subset \partial \mathbb{D}$ ?

Problem 8.4 (Dirichlet energy of $z$ ). In the bounded degree case, by applying the main theorem of [2], convergence to the boundary may be shown by observing that the Dirichlet energy of the centres function $z$ is finite:

$$
\mathcal{E}(z)=\sum_{u \sim v}(z(u)-z(v))^{2} \leq \sum 2 \operatorname{deg}(v) r(v)^{2} \leq 2 \max \{\operatorname{deg}(v)\}
$$

Is the Dirichlet energy of $z$ almost surely finite for a unimodular random rooted CP hyperbolic triangulation? This may provide a route to weakening the moment assumption in our results.

Problem 8.5 (Other embeddings). How does the canonical embedding of the Poisson-Delaunay triangulation differ from the embedding given by the circle packing? Is there a circle packing so that $d_{h y p}\left(v, z_{h}(v)\right)$ is stationary?

The conformal embedding of a triangulation is defined by forming a Riemann surface by gluing equilateral triangles according to the combinatorics of the triangulation before mapping the resulting surface conformally to $\mathbb{D}$ or $\mathbb{C}$. Is it possible to control the large scale distortion between the conformal embedding and the circle packing? In general the answer is no, but in the unimodular case there is hope.

Regardless of the answer to this question, our methods should extend without too much difficulty to establish analogues of Theorem 1.3 for these other embeddings, the main obstacle being to show almost sure convergence of the random walk to a point in the boundary $\partial \mathbb{D}$.

Problem 8.6. Reduce the moment assumption on $\operatorname{deg}(\rho)$ in Theorems 1.3 and 1.4. Finite expectation is needed to switch to a reversible distribution on rooted maps, but perhaps the second moment is not needed. 


\section{Acknowledgments}

OA is supported in part by NSERC. AN is supported by the Israel Science Foundation grant 1207/15 as well as NSERC and NSF grants. GR is supported in part by the Engineering and Physical Sciences Research Council under grant $\mathrm{EP} / 103372 \mathrm{X} / 1$.

All circle packings above were generated using Ken Stephenson's CirclePack software [40]. We thank Ken for his assistance using this software and for useful conversations. We also thank the referee for their comments and suggestions.

\section{References}

[1] D. Aldous and R. Lyons. Processes on unimodular random networks. Electron. J. Probab., 12:no. 54, 1454-1508, 2007.

[2] A. Ancona, R. Lyons, and Y. Peres. Crossing estimates and convergence of Dirichlet functions along random walk and diffusion paths. Ann. Probab., 27(2):970-989, 1999 .

[3] O. Angel. Growth and percolation on the uniform infinite planar triangulation. Geom. Funct. Anal., 13(5):935-974, 2003.

[4] O. Angel, M. T. Barlow, O. Gurel-Gurevich, and A. N. s. Boundaries of planar graphs, via circle packings. Ann. Probab., to appear, arXiv:1311.3363.

[5] O. Angel, G. Chapuy, N. Curien, and G. Ray. The local limit of unicellular maps in high genus. Elec. Comm. Prob, 18:1-8, 2013.

[6] O. Angel and N. Curien. Percolations on random maps I: Half-plane models. Ann. Inst. Henri Poincaré Probab. Stat., 51(2):405-431, 2015.

[7] O. Angel, T. Hutchcroft, A. Nachmias, and G. Ray. Hyperbolic and parabolic unimodular random maps. In preparation.

[8] O. Angel, A. Nachmias, and G. Ray. Random walks on stochastic hyperbolic half planar triangulations. Random Structures Algorithms. To appear. arXiv:1408.4196.

[9] O. Angel and G. Ray. Classification of half-planar maps. Ann. Probab., 43(3):13151349, 2015.

[10] O. Angel and O. Schramm. Uniform infinite planar triangulations. Comm. Math. Phys., 241(2-3):191-213, 2003.

[11] A. F. Beardon and K. Stephenson. Circle packings in different geometries. Tohoku Math. J. (2), 43(1):27-36, 1991. 
[12] I. Benjamini and N. Curien. Ergodic theory on stationary random graphs. Electron. J. Probab., 17:no. 93, 20, 2012.

[13] I. Benjamini and N. Curien. Simple random walk on the uniform infinite planar quadrangulation: subdiffusivity via pioneer points. Geom. Funct. Anal., 23(2):501$531,2013$.

[14] I. Benjamini, R. Lyons, and O. Schramm. Percolation perturbations in potential theory and random walks. In Random walks and discrete potential theory (Cortona, 1997), Sympos. Math., XXXIX, pages 56-84. Cambridge Univ. Press, Cambridge, 1999.

[15] I. Benjamini, E. Paquette, and J. Pfeffer. Anchored expansion, speed, and the hyperbolic Poisson Voronoi tessellation. ArXiv e-prints, Sept. 2014.

[16] I. Benjamini and O. Schramm. Harmonic functions on planar and almost planar graphs and manifolds, via circle packings. Invent. Math., 126(3):565-587, 1996.

[17] I. Benjamini and O. Schramm. Random walks and harmonic functions on infinite planar graphs using square tilings. Ann. Probab., 24(3):1219-1238, 1996.

[18] I. Benjamini and O. Schramm. Percolation in the hyperbolic plane. J. Amer. Math. Soc., 14(2):487-507 (electronic), 2001.

[19] I. Benjamini and O. Schramm. Recurrence of distributional limits of finite planar graphs. Electron. J. Probab., 6:no. 23, 1-13, 2001.

[20] N. Curien. Planar stochastic hyperbolic triangulations. Probability Theory and Related Fields, pages 1-32, 2015.

[21] N. Curien and G. Miermont. Uniform infinite planar quadrangulations with a boundary. Random Structures Algorithms, 47(1):30-58, 2012.

[22] H. Furstenberg. A Poisson formula for semi-simple Lie groups. Ann. Math., $77(2): 335-386,1963$.

[23] A. Georgakopoulos. The boundary of a square tiling of a graph coincides with the poisson boundary. Invent. Math. To appear.

[24] O. Gurel-Gurevich and A. Nachmias. Recurrence of planar graph limits. Ann. of Math. (2), 177(2):761-781, 2013.

[25] L. J. Hansen. On the Rodin and Sullivan ring lemma. Complex Variables Theory Appl., 10(1):23-30, 1988.

[26] A. Hatcher. Algebraic topology. Cambridge University Press, Cambridge, 2002.

[27] Z.-X. He and O. Schramm. Fixed points, Koebe uniformization and circle packings. Ann. of Math. (2), 137(2):369-406, 1993. 
[28] Z.-X. He and O. Schramm. Hyperbolic and parabolic packings. Discrete Comput. Geom., 14(2):123-149, 1995.

[29] V. A. Kaimanovich. Measure-theoretic boundaries of Markov chains, 0-2 laws and entropy. In Proceedings of the Conference on Harmonic Analysis and Discrete Potential Theory (Frascati, pages 145-180. Plenum, 1991.

[30] H. Kesten. Symmetric random walks on groups. Trans. Amer. Math. Soc., 92:336$354,1959$.

[31] P. Koebe. Kontaktprobleme der konformen Abbildung. Hirzel, 1936.

[32] R. J. Lipton and R. E. Tarjan. Applications of a planar separator theorem. SIAM J. Comput., 9(3):615-627, 1980.

[33] R. Lyons and Y. Peres. Probability on Trees and Networks. Cambridge University Press, 2015. In preparation. Current version available at http://mypage.iu.edu/〜rdlyons/.

[34] G. L. Miller, S.-H. Teng, W. Thurston, and S. A. Vavasis. Separators for spherepackings and nearest neighbor graphs. J. ACM, 44(1):1-29, Jan. 1997.

[35] G. Pete. Probability and geometry on groups. http://www.math.bme.hu/ gabor/PGG.pdf, 2014.

[36] G. Ray. Hyperbolic random maps. PhD thesis, UBC, 2014.

[37] B. Rodin and D. Sullivan. The convergence of circle packings to the Riemann mapping. J. Differential Geom., 26(2):349-360, 1987.

[38] S. Rohde. Oded Schramm: From Circle Packing to SLE. Ann. Probab., 39:1621-1667, 2011.

[39] O. Schramm. Rigidity of infinite (circle) packings. J. Amer. Math. Soc., 4(1):127-149, 1991.

[40] K. Stephenson. Circle pack, java 2.0. http:/www . math.utk. edu/ ^kens/CirclePack.

[41] K. Stephenson. Introduction to circle packing. Cambridge University Press, Cambridge, 2005. The theory of discrete analytic functions.

[42] W. P. Thurston. The geometry and topology of 3-manifolds. Princeton lecture notes., 1978-1981.

[43] B. Virág. Anchored expansion and random walk. Geom. Funct. Anal., 10(6):1588$1605,2000$.

[44] W. Woess. Random walks on infinite graphs and groups, volume 138 of Cambridge Tracts in Mathematics. Cambridge University Press, Cambridge, 2000. 
Omer Angel, Tom Hutchcroft

Department of Mathematics, University of British Columbia

Email: $\{$ angel, thutch\}@math.ubc.ca

Asaf Nachmias

School of Mathematical Sciences, Tel Aviv University

Email: asafnach@post.tau.ac.il

Gourab Ray

Statistical Laboratory, University of CAMbridge

Email: rg508@cam.ac.uk 